\title{
Die Bestimmung des notwendigen Lebensstandards - Einschätzungsunterschiede und Entscheidungsprobleme
}

\author{
Gero Lipsmeier ${ }^{1}$
}

Universität Bielefeld, Fakultät für Soziologie, Postfach 1001 31, D-33501 Bielefeld

Zusammenfassung: Der Beitrag behandelt konzeptionelle und empirische Aspekte der empirischen Bestimmung des notwendigen Lebensstandards mit repräsentativen Umfragedaten für Deutschland. Dabei wird der Frage nachgegangen, aus welchen Gründen und in welchem Ausmaß Personen in unterschiedlichen Lebenssituationen (u.a. Haushaltstypen, Alter, Bildung regionale Merkmale) heterogene Ansichten über die Bedeutung einer breit angelegten Liste von Lebensstandardmerkmalen vertreten. Es wird mit multivariaten Methoden gezeigt, daß sich diese Einschätzungen nur für eine begrenzte Auswahl von Merkmalen in nennenswertem Ausmaß unterscheiden. Demgegenüber variiert die Bewertung der Notwendigkeit deutlich mit der Verbreitung der Merkmale in der Gesellschaft und mit der individuellen Verfügbarkeit. Für die Messung der Teilhabe am Lebensstandard mit Deprivationsindizes kann gezeigt werden, daß unterschiedliche Varianten der Berechnung zu Maßen führen, die extrem hoch miteinander korrelieren. Daraus wird der Schluß abgeleitet, daß auf eine Berücksichtigung von ohnehin geringen Bewertungsunterschieden durch gewichtete Deprivationsindizes verzichtet werden kann.

\section{Einleitung und Problemstellung}

Nach der Definition des Rates der Europäischen Gemeinschaften vom 19.12.1984 werden Einzelpersonen, Familien und Personengruppen als arm bezeichnet, wenn sie „... über so geringe (materielle, kulturelle und soziale) Mittel verfügen, daß sie von der Lebensweise ausgeschlossen sind, die in dem Mitgliedsstaat in dem sie leben, als Minimum annehmbar ist" (Europäische Gemeinschaften1985: 24).

Die empirische Bestimmung von gesellschaftlichen (Teil-)Gruppen oder Individuen, die innerhalb einer Gesellschaft als arm zu betrachten sind, ist nicht zuletzt deshalb ein so schwieriges und kontrovers diskutiertes Unterfangen, weil die Definition von Mindestniveaus unausweichlich normative Setzungen erfordert. Veit-Wilson (1998: 12ff) betont in diesem Zusammenhang, daß es wichtig ist zu unterscheiden durch wen und mit welchen Zielen und Legitimationen derartige Setzungen erfolgen. Dabei weist er den Sozialwissenschaften - in Abgrenzung zum präskriptiven, über Macht legitimierten Vorgehen der Politik - die Aufgabe zu, die in einer Gesellschaft insgesamt

\footnotetext{
${ }^{1}$ Die in diesem Beitrag berichteten Forschungen wurden durch einen vom Autor gemeinsam mit Hans-Jürgen Andreß eingeworbenen Studienpreis, den GFM-GETAS/ WBA anläßlich seines 50jährigen Jubiläums gestiftet hat und aus Mitteln des Ministeriums für Wissenschaft und Forschung des Landes Nordrhein-Westfalen gefördert. Für wertvolle Hinweise und Anregungen danke ich HansJurgen Andreß, Stefan Hirschauer und zwei anonymen Gutachtern.
}

vorherrschende Definition zu erforschen. In diesem Beitrag geht es um Voraussetzungen und mögliche Probleme der empirischen Bestimmung eines minimal notwendigen Lebensstandards mit Umfragedaten für Deutschland. Das hier verfolgte Vorgehen orientiert sich an verschiedenen Vorschlägen zur Messung von Armut in der Tradition des von Townsend (1979) vorgeschlagen Ansatzes relativer Deprivation. Von zentraler Bedeutung ist dabei die erstmals von Mack und Lansley (1985) eingebrachte Erweiterung des empirischen Vorgehens von Townsend um die Einbeziehung subjektiver Bewertungen der Notwendigkeit verschiedener Merkmale des alltäglichen Lebens für einen minimalen Lebensstandard durch Befragte einer bevölkerungsweiten Umfrage. Die Berücksichtigung derartiger Bewertungen erfolgt in der erklärten Absicht, die normative Entscheidung darüber, welche Aspekte und Merkmale des alltäglichen Lebens als Indikatoren für eine minimal akzeptable Lebensweise herangezogen werden, möglichst wenig in den Händen von (wissenschaftlichen) Beobachtern zu belassen: „[This study] aims to identify a minimum acceptable way of life not by reference to the view of ,experts', nor by reference to observed patterns of expenditure or observed living standards, but by reference to the views of society as a whole. This is, in essence, a consensual approach to defining minimum standards." (Mack/ Lansley 1985: 42, Hervorhebungen im Original). ${ }^{2}$

\footnotetext{
${ }^{2}$ Aus einer stärker ökonomisch orientierten Forschungsrichtung wurden mit unterschiedlichen Verfahren ähnliche Ziele bei der Bestimmung von Einkommensgrenzen verfolgt, die durch Umfrageergebnisse gewonnenen wur-
} 
Eine Gesellschaft als ganzes kann keine Ansichten haben. Insofern erfordert die Bestimmung eines gesellschaftlichen Konsenses die Auseinandersetzung mit den Sichtweisen von Individuen. Von einem Konsens würde man dann sprechen können, wenn alle Mitglieder der Gesellschaft die gleichen Ansichten über den minimal notwendigen Lebensstandard vertreten würden. Das erscheint in hochdifferenzierten modernen Gesellschaften zumindest dann als unwahrscheinlich, wenn Lebensstandarddimensionen bewertet werden sollen, die über ein physisches Existenzminimum hinausgehen. Das empirische Vorgehen von Mack und Lansley zielt deshalb auch nicht auf eine derart weitreichende Übereinstimmung der Sichtweisen, sondern auf die Bestimmung der Dimensionen und Merkmale der alltäglichen Lebensweise, die eine Mehrheit der Bevölkerung zum notwendigen Lebensstandard rechnet. Insofern ist die Bezeichnung dieses Ansatzes als ,konsensuell' irreführend. Das Ausmaß der Übereinstimmung bzw. der Heterogenität von im Interview durch Personen aus unterschiedlichen Bevölkerungsgruppen geäußerten Notwendigkeitsbewertungen bedarf deshalb einer genaueren Untersuchung. Mack und Lansley (1985) konnten ähnlich wie Gordon/Pantazis (1997a) und Nolan/Whelan (1996) eine weitgehende Übereinstimmung der Sichtweisen zeigen und betonen die grundlegende Bedeutung dieser Feststellung für den von ihnen verfolgten Ansatz. Demgegenüber heben Halleröd (1994), Halleröd et al. (1997) und van den Bosch (1998) deren Heterogenität hervor, wenn nach verschiedenen soziodemographischen Merkmalen differenziert wird. In Erweiterung bislang vorliegender Ergebnisse für Deutschland (Andreß/Lipsmeier 1995, Lipsmeier 1995, Andreß et al. 1996) soll dieser zentralen Frage hier differenzierter und auf der Basis einer größeren und besser verallgemeinerbaren $\mathrm{Da}$ tenbasis nachgegangen werden.

Vor dem Hintergrund der insbesondere von Halleröd (1994) hervorgehobenen unterschiedlichen Ansichten verschiedener Bevölkerungsgruppen zur Bedeutung einiger Aspekte des Lebensstandards wurde wiederholt diskutiert (u.a. Halleröd 1994, 1995; Nolan/Whelan 1996, van den Bosch 1998), inwieweit der Versuch, einen Mindeststandard für eine ganze Gesellschaft zu definieren, sinnvoll ist. Die Kritiker betonen dabei in der Regel die Bedeutung von Referenzgruppen für die

den (u.a.: Goedhart et al. 1977, Hagenaars 1986). Diese Vorschläge werden in der einschlägigen Literatur z.T. als „Konsensuelle Einkommensgrenzen" bezeichnet.
Bestimmung der Dimensionen und Merkmale, die als Minimum akzeptabel sind. Nach welchen Kriterien sich soziologisch relevante Vergleichsgruppen identifizieren lassen und welche Vergleichsdimensionen in diesem Zusammenhang von Bedeutung sind, wurde bislang jedoch kaum systematisch untersucht. Es liegen verschiedene Vorschläge dazu vor, wie referenzgruppenspezifische Mindeststandards bei der Messung der Teilhabe hieran durch geeignet konstruierte Deprivationsindizes berücksichtigt werden können (u.a. Desai/Shah 1988; Muffels 1993; Halleröd 1994). Dabei fehlt jedoch weitgehend eine systematische Diskussion der (sozialpolitischen) Konsequenzen für die Interpretation von Deprivationsindizes, wenn durch die Einbeziehung unterschiedlicher Mindeststandards keine gemeinsame Referenz verfügbar ist.

Unabhängig von der Berücksichtigung von Referenzgruppen besteht auch keine Einigkeit in der Frage, ob allen erhobenen Lebensstandardmerkmalen im Falle ihres (durch unzureichende Ressourcen) erzwungenen Fehlens die gleiche substantielle Bedeutung für das Ausma $B$ der Teilhabe am Mindeststandard beigemessen werden soll. Mack und Lansley (1985) sowie Nolan und Whelan (1996) betrachten die fehlende Teilhabe an einem der mehrheitlich zum minimal notwendigen Standard gerechneten Merkmale unabhängig von weiteren Bedingungen als Indikator für Deprivation. Demgegenüber wurde auch argumentiert, daß Merkmale, die in der Gesellschaft bzw. Referenzgruppe weit verbreitet sind, mit einem höheren Gewicht in Deprivationsmaße eingehen sollten als seltene (Luxus-)Merkmale (Desai/Shah 1988, Muffels 1993), da sie einen stärkeren Beitrag zu Deprivation leisten, wenn ein Individuum nicht über sie verfügen kann. Halleröd (1994) und van den Bosch (1998) argumentieren schließlich für eine Gewichtung mit dem Anteil von Befragten, die das jeweilige Merkmal für notwendig halten. Sowohl für Deutschland als auch international fehlt bislang weitgehend eine vergleichende $\mathrm{Ge}$ genüberstellung verschiedener Deprivationsindizes. Die vorliegenden Anwendungen dieser Verfahren für die substantielle Armutsforschung greifen in der Regel auf nur einen Index zurück. Aus diesem Grund möchte ich hier auch einen empirischen Beitrag zur Klärung der Frage leisten, ob bei verschiedenen Varianten der Indexbildung mit unterschiedlichen substantiellen Ergebnissen zu rechnen ist.

Bereits die Erhebung der Ansichten über den notwendigen Lebensstandard mit Mitteln der Umfrageforschung ist zudem durchaus voraussetzungs- 
reich. Das Ziel der Erhebung sollten nicht die individuellen Aspirationen und Wünsche von Befragten sein, sondern deren normative Einschätzung der Bedeutung von Lebensstandardmerkmalen für einen ausreichenden Lebensstandard. Erhebungstechnisch ist daran problematisch, da $B$ diese beiden Aspekte kaum unabhängig voneinander sein dürften. Selbst wenn man in einer Befragung also explizit auf die Bewertung für einen allgemein notwendigen Lebensstandard abstellt, dürfte es schwierig sein, einen empirischen Nachweis dafür zu erbringen, daß die Befragten tatsächlich auf ein vergleichsweise abstraktes Konstrukt wie den allgemeinen Lebensstandard rekurrieren. In eine ähnliche Richtung weist der Einwand von Walker (1987: 219), daß vermutlich viele Menschen ihr Urteil über die Bedeutung von Lebensstandardmerkmalen ändern würden, wenn sie mehr Informationen über den Lebensstandard und die Bewertungen durch andere erhielten. An diesen Einwand schließt sich das erhebungstechnische Problem der Frageformulierung und der Verbalisierung von Antwortvorgaben an. Es ist zu erwarten, daß Einschätzungen zur allgemeinen Bedeutung von Lebensstandardmerkmalen nicht als fest verankerte, dichotome Bewertungen im Bewußtsein von potentiellen Befragten vorliegen. Insofern erscheint es als sinnvoll, Abstufungen in der Notwendigkeitseinschätzung bereits bei der Erhebung zuzulassen. Damit wird eine Abschätzung der Sicherheit möglich, mit der verschiedene Aspekte des Lebensstandards als notwendiger Bestandteil eines Mindeststandards gesehen werden. Die bislang vorliegenden Studien aus verschiedenen europäischen Ländern (u.a. Mack/Lansley 1985, Muffels 1993, Halleröd 1995, Nolan/Whelan 1996, Gordon/Pantazis 1997b, van den Bosch 1998, Kangas/ Ritakallio 1998) verwenden demgegenüber durchgängig eine dichotome Abfrage der Notwendigkeitseinschätzung.

Den vorstehend umrissenen Forschungsfragestellungen wird hier aus der Perspektive nachgegangen, inwieweit und in welchen Bereichen die Übertragung des Ansatzes von Mack und Lansley auf deutsche Verhältnisse mit ähnlichen Problemen konfrontiert ist, wie sie für andere europäische Länder diskutiert werden. Dabei wird zunächst zu untersuchen sein, welche Dimensionen und Merkmale des alltäglichen Lebens von deutschen Befragten zum notwendigen Lebensstandard gerechnet werden. Neben dem Zusammenhang zwischen Verbreitung und Notwendigkeitsbewertung sowie zwischen individueller Verfügbarkeit und dieser Bewertung wird dabei vor allem zu prüfen sein, wie homogen oder heterogen die Ansichten verschiedener gesellschaftlicher (Teil-)Gruppen sind. Speziell für Deutschland ist dabei auch die Frage nach eventuell unterschiedlichen Vorstellungen zwischen den alten und den neuen Bundesländern von Interesse. Abschließend möchte ich untersuchen, ob sich verschiedene Varianten von Deprivationsindizes in nennenswertem Umfang unterscheiden, wenn man sie mit Daten für Deutschland implementiert.

Im folgenden 2. Abschnitt werde ich zunächst das eingesetzte Erhebungsinstrument mit den erhobenen Lebensstandarddimensionen vorstellen. Daran anschließend (3.) werden einige Hypothesen und ein Analysemodell für die zu erwartenden Einflüsse auf die Bewertung der Notwendigkeit entwickelt. Nach einer Darstellung der empirischen Ergebnisse im 4. Abschnitt beschlieBt eine zusammenfassende Diskussion den Beitrag.

\section{Das Erhebungsinstrument}

Den im folgenden berichteten Forschungsergebnissen liegt eine Einschaltung in den von ZUMA e.V. und GFM-Getas/WBA gemeinsam durchgeführten Sozialwissenschaftenbus III/1996 zugrunde. Befragt wurden in standardisierten, mündlichen Interviews insgesamt 3.170 Personen. Hiervon lebten zum Zeitpunkt der Befragung 1.989 in den alten Bundesländern und 1.181 in den neuen Bundesländern. ${ }^{3}$

Mit zwei Blöcken von jeweils 42 Fragen wurden zum einen Notwendigkeitseinschätzungen und zum anderen die individuelle Verfügbarkeit über einen bzw. die Partizipation an einem weiten Bereich von Merkmalen des alltäglichen Lebensstandards erhoben. Die Befragungspersonen wurden im ersten Block zunächst gebeten, eine Auswahl von Konsumgütern, Ausstattungsmerkmalen und sozialen Aktivitäten daraufhin zu bewerten, inwieweit diese zur Erreichung bzw. Sicherstellung eines normalen, ausreichend guten Lebensstandards für alle Menschen und Haushalte in Deutschland notwendig sind. Dabei waren vier Antwortmöglichkeiten von ,unbedingt notwendig ' bis ,überhaupt nicht notwendig' vorgegeben. Wenn im folgenden von den Ansichten der Befragten zum minimal notwendigen Lebensstandard die Rede sein wird, so beziehe ich mich dabei ausschließlich auf die

\footnotetext{
${ }^{3}$ Weitere Einzelheiten zur Stichprobe und zur technischen Durchfuhrung der Studie finden sich in GFM-Getas/WBA 1997.
} 
Nennung ,unbedingt notwendig'. Demgegenüber umfaßt die Bezeichnung als notwendiger Lebensstandard die zusammengefaßten Antworten ,unbedingt notwendig' und ,eher notwendig'.

Vier Items beziehen sich ausdrücklich auf den Lebensstandard von Familien mit Kindern. In der Frageformulierung wurden die Befragten dabei aufgefordert, an den notwendigen Lebensstandard von Familien mit Kindern zu denken. Ebenso wurde die Bewertung der Notwendigkeit für vier Arbeitsplatzmerkmale erhoben. Dabei waren die Befragten aufgefordert, an den notwendigen Lebensstandard von Erwerbstätigen und Arbeit Suchenden zu denken. Abschließend sollten noch 7 Infrastrukturmerkmale daraufhin bewertet werden, inwiefern es zum notwendigen Lebensstandard gehört, daß sich diese in der Nähe, $d . h$. nicht weiter als 15 Minuten zu Fuß von der Wohnung entfernt befinden. Es ist wichtig zu betonen, daß bewußt nicht gefragt wurde, was die Personen für sich persönlich oder ihren Haushalt für erstrebenswert oder wünschbar halten. Ziel war es, ein im wesentlichen normatives Urteil über die Bedeutung der erfragten Aspekte des Lebensstandards in Deutschland zu erhalten.

Im zweiten Fragenblock wurde für die gleichen Items gefragt, ob die Befragungspersonen persönlich oder ihr Haushalt über diese Dinge verfügen bzw. die genannten Tätigkeiten ausüben. Unter Armutsgesichtspunkten interessiert jedoch nicht der freiwillige Verzicht auf einzelne Items z. B. des Vegetariers auf Mahlzeiten mit Fleisch oder der Umweltbewußten auf ein Auto, sondern die $\boldsymbol{U n}$ möglichkeit, sich bestimmte Dinge oder Aktivitäten leisten zu können. Das Fehlen von Merkmalen des Lebensstandards ist nur dann ein Indikator für Armut im Sinne der eingangs zitierten Definition, wenn es durch unzureichende Ressourcen verursacht wurde. Deshalb wurde in den Antwortvorgaben nach den Gründen für das eventuelle Fehlen von Items differenziert. Vorgegeben waren die Antwortmöglichkeiten ,ja, , nein, ist aus finanziellen Gründen nicht möglich' und ,nein, trifft aus anderen Gründen nicht zu'.

Die erfragten Lebensstandardmerkmale decken verschiedene Dimensionen ab: Ernährung (Items E1 - E3), persönlicher Bedarf und Haushaltsausstattung (P1 - P8), Wohnumfeld und Wohnungsausstattung (W1 - W6), Zahlungsfähigkeit und finanzielle Rücklagen ( $\mathrm{Z1}-\mathrm{Z3})$, Bildungs- (B1) und Freizeitaktivitäten (F1 - F3), Sozialkontakte (S1, S2) sowie die Gesundheitsvorsorge (G1). Weiterhin wurden Merkmale des Lebensstandards von
Kindern (K1 - K4), des Arbeitsplatzes (A1 - A4) und der Infrastruktur (I1 - I7) einbezogen. Die Auswahl der Items orientiert sich zum einen an ähnlichen Fragenkomplexen aus Umfragen in anderen europäischen Ländern (für einen Überblick vgl. Whelan 1993), zum anderen basiert sie auf Erfahrungen mit einer früheren Umfrage in Deutschland (Andreß 1994; AndreB/ Lipsmeier 1995; AndreB et al. 1996). Bis zu einem gewissen Grad ist die Entscheidung für oder gegen die Aufnahme konkreter Items von der subjektiven Einschätzung der Forscher und Forscherinnen abhängig und der eine oder die andere mag einzelne Merkmale vermissen oder andere weniger zentral finden. Die Liste von möglichen Indikatoren für den Lebensstandard in Deutschland ist prinzipiell nahezu unendlich. Es kann deshalb nicht darum gehen, alle denkbaren konkreten Güter oder Tätigkeiten in das Erhebungsinstrument aufzunehmen. Wesentlich ist vielmehr, Items zu finden, welche die wesentlichen Bereiche von Lebensweisen hinreichend abdecken (Townsend 1979: 249). Die oben angesprochene frühere Untersuchung enthielt eine offene Frage, in der nach weiteren Lebensstandardmerkmalen gefragt wurde, die aus der Sicht der Befragten zum notwendigen Lebensstandard zu rechnen seien. Eine Auswertung dieser Frage (Lipsmeier 1995) führte zu keinen deutlichen Hinweisen auf wesentliche Lebensstandarddimensionen, die im damals verwendeten und hier erweiterten Instrument fehlen.

\section{Fragestellungen, Erklärungsansätze und Hypothesen}

Befragt man eine Stichprobe von Personen dazu, inwieweit sie eine Auswahl von Merkmalen des alltäglichen Lebens für notwendig halten „wenn man in Deutschland für alle Menschen einen normalen, ausreichend guten Lebensstandard erreichen bzw. sicherstellen wollte", so stellt sich die Frage, wovon derartige Urteile und eventuelle Bewertungsunterschiede abhängen. Vier Aspekte scheinen hier von Bedeutung zu sein: 1. Für welche Items oder Itemgruppen ist mit nennenswerten Unterschieden in der Bewertung zu rechnen? 2. Welche Rolle spielt die (wahrgenommene) Verbreitung von Lebensstandardmerkmalen? 3. Lassen sich im Antwortverhalten Hinweise darauf finden, da $B$ es mit der gewählten Fragestellung gelungen ist, die Befragten zur Einnahme einer allgemeinen (eben normativen) Perspektive zu bewegen? und worin sind (4.) Gründe für eine ggf. 
unterschiedliche Bewertung von Items durch bestimmte gesellschaftliche Gruppen zu vermuten.

In Hinblick auf den ersten Punkt erscheint es sinnvoll, zunächst solche Merkmale zu identifizieren, für die überhaupt mit nennenswerten Unterschieden - und damit theoretischem und empirischem Erklärungsbedarf - zu rechnen ist. In einer vergleichsweise reichen Gesellschaft wie Deutschland ist wohl davon auszugehen, daß es kaum Uneinigkeit darüber geben wird, daß Merkmale des Subsistenzbedarfs (z. B. ausreichende Kleidung, angemessener Wohnraum, ausreichende Ernährung) zum notwendigen Lebensstandard $\mathrm{zu}$ rechnen sind.

$\mathrm{H}_{1}$ : Es gibt einen Kern von Lebensstandardmerkmalen (Grundbedarfsmerkmale), die von einer so großen Mehrheit der Befragten zum notwendigen Lebensstandard gerechnet werden, daß faktisch von einem gesellschaftlichen Konsens über die Notwendigkeit dieser Merkmale ausgegangen werden kann.

Wenn sich eine solche Gruppe von Lebensstandardmerkmalen identifizieren läßt, so ist davon auszugehen, daß diese von einem ebenfalls sehr großen Teil der Befragten nicht nur als, eher notwendig' sondern als , unbedingt notwendig' bewertet werden. Für Merkmale, die über den Subsistenzbedarf hinausgehen, ist demgegenüber zu erwarten, daß sich diese Abgrenzung zu Merkmalen eines erweiterten Bedarfs neben einer möglicherweise größeren Heterogenität der Bewertungen auch darin wiederspiegelt, daß letztere deutlich seltener als Bestandteil des minimal notwendigen Lebensstandards genannt werden.

Für die subjektive Bewertung der Gerechtigkeit von Einkommensverteilungen und die Schätzung der Höhe von ,gerechten ' Einkommen existiert umfangreiche Forschungsliteratur. Dort wird u.a. die Bedeutung von persönlichen und sozialen Werten (Dickinson 1991, Wegener/Liebig 1993), von sozialen Vergleichen (Alves/Rossi 1978; Dornstein 1989) und die Perzeption von unterschiedlichen Bedarfslagen (Lamm/Schwinger 1980) diskutiert. Eine gewisse Nähe der von dieser Forschungsrichtung untersuchten Gerechtigkeitsurteile zu der hier interessierenden Bewertung des notwendigen Lebensstandards liegt bereits aufgrund des in beiden Fällen normativen Charakters der abzugebenden Urteile (Was steht anderen Menschen als Minimum zu?) nahe. Ohne die ebenfalls deutlichen Unterschiede - insbesondere den dort vorherrschenden Fokus auf Einkommen und die zu erwartende Unterstützung für Einkommensumverteilungen - zu verleugnen, lassen sich m.E. einige Er- klärungsansätze und empirische Ergebnisse der Gerechtigkeitsforschung zur Herleitung von $\mathbf{H y}-$ pothesen über zu erwartende Einflüsse auf die Bewertung von Lebensstandardmerkmalen heranziehen.

So konnte z. B. Dickinson (1991) in Übereinstimmung mit der von Hochschild (1981: 48ff) postulierten Bereichsabhängigkeit von Bewertungsprinzipien feststellen, daß Gerechtigkeitsurteile weniger mit festen Wertüberzeugungen (Rokeach 1973) als mit Bewertungsprinzipien zusammenhängen, die an die jeweils zu bewertende Situation angepaßt werden. Daraus läßt sich für die Bewertung von verschiedenen Lebenstandarddimensionen die Vermutung ableiten, $\mathrm{da} \beta$ für Items mit unterschiedlichen Charakteristika auch unterschiedliche Bewertungsprinzipien herangezogen werden. Je nach zu bewertender Dimension (z. B. Ernährung vs. Freizeitverhalten) werden unterschiedliche Bewertungsprinzipien (z. B. Gleichheit, Bedarf) eine unterschiedliche Relevanz haben.

Alves und Rossi (1978) konnten zeigen, daß die Höhe des als gerecht bewerteten Einkommens systematisch mit der vom Bewerter wahrgenommenen Einkommensverteilung variiert. Was von den Mitgliedern einer Gesellschaft zum notwendigen oder minimal notwendigen Lebensstandard gerechnet wird, hängt vermutlich ebenfalls recht eng mit der wahrgenommenen Verbreitung der jeweiligen Merkmale zusammen: “... those styles of living that are widespread are equivalent to those that are socially approved, encouraged or expected" (Mack/Lansley 1985: 67).

$\mathrm{H}_{2}$ : Merkmale, die in der gesamten Gesellschaft weit verbreitet sind, werden in der Regel auch von vielen Menschen zum notwendigen Lebensstandard gerechnet.

Während die grundsätzliche Richtung dieses $\mathrm{Zu}$ sammenhanges auch für den minimal notwendigen Lebensstandard zu erwarten ist, so ist er vermutlich weniger eng.

Wenn es gelungen ist, normative Überzeugungen zum notwendigen Lebensstandard in Deutschland zu erheben, sollten sich im Antwortverhalten der Befragten parallel und im teilweisen Widerspruch $\mathrm{zu} \mathrm{H}_{2}$ Hinweise darauf finden lassen, da $\beta$ die $\mathrm{Be}-$ fragten nicht einfach Notwendigkeitsbewertung und (wahrgenommene) Verbreitung gleichsetzen. Es sollten sich also bereits auf der Ebene der Anteilswerte für die gesamte Stichprobe bei verschiedenen Merkmalen Unterschiede feststellen lassen zwischen dem Anteil von Befragten, die über das jeweilige Merkmal verfügen und dem Anteil der Befragten, die dieses zum notwendigen Lebens- 
standard rechnen. Dabei sind für unterschiedliche Bereiche des Lebensstandards Abweichungen der Notwendigkeitsbewertungen von der Verbreitung in zwei Richtungen erwartbar:

$\mathrm{H}_{3}$ : Bei Merkmalen, deren individuelle Verfügbarkeit stärker von strukturellen Faktoren als von individuellen Entscheidungen abhängt (z. B. Arbeitsplatzmerkmale, Wohnraum, Infrastruktur), ist zu erwarten, daß diese von einem sehr hohen Anteil der Befragten zum notwendigen Lebensstandard gerechnet werden, obwohl möglicherweise nur ein deutlich geringerer Anteil der Befragten über diese Merkmale verfügt.

Das mag insbesondere für Arbeitsplatz- und Wohnungsmerkmale in den neuen Bundesländern zutreffen.

$\mathrm{H}_{4}$ : Bei anderen Merkmalen - insbesondere solchen, die nicht zum Grundbedarf zu rechnen sind - ist zu erwarten, $\mathrm{daB}$ diese in einem wohlhabenden Land wie Deutschland zwar weit verbreitet sind, jedoch von einem deutlich geringeren Teil der Bevölkerung zum notwendigen - und erst recht zum minimal notwendigen - Lebensstandard gerechnet werden.

Der in $\mathrm{H}_{2}$ unterstellte Zusammenhang setzt u.a. voraus, daß die Menschen über die faktische Verbreitung dieser Merkmale in der Gesellschaft gut informiert sind oder zumindest subjektiv davon überzeugt sind den Verbreitungsgrad zu kennen. Für die in $\mathbf{H}_{1}$ als Grundbedarf bezeichneten Merkmale wird man wohl plausibel unterstellen können, daß über eine sehr weite Verbreitung kaum Unsicherheit besteht. Insofern ließe sich ein weitgehender Konsens über die hohe Bedeutung dieser Merkmale für den notwendigen Lebensstandard auch auf die wahrgenommene oder angenommene hohe Verbreitung zurückführen.

Merkmale, bei denen keine so große Einigkeit der Bevölkerung über deren Notwendigkeit beobachtet wird, sind demgegenüber möglicherweise gerade dadurch gekennzeichnet, daß auch die Unsicherheit über deren Verbreitung größer ist. Ein Grund für diese Vermutung ist die Tatsache, daß die erfragten Lebensstandardmerkmale in ganz unterschiedlichem Ausmaß Gegenstand öffentlicher Diskurse sind. Vergleichsweise häufig sind z. B. die Lebensbereiche Arbeit und Gesundheit als Ziel (sozial-)politischer Intervention Gegenstand der Berichterstattung in den Medien. Demgegenüber werden die Bereiche des individuellen Konsums und der Freizeitgestaltung wesentlich seltener thematisiert. Insofern ist eine breite Informiertheit über die tatsächliche Verbreitung nicht umstandslos für alle Merkmale im gleichen Ausmaß zu unterstellen. Öffentliche Diskurse über einzelne Bereiche des Lebensstandards führen nicht nur zu einer unterschiedlichen Informiertheit über die Verteilung dieser Bereiche in eine Gesellschaft. Ebenso ist zu erwarten, daß die individuelle Bewertung der Bedeutung von Lebensstandardmerkmalen direkt durch die in öffentlichen Diskursen vermittelte Bedeutung z. B. für sozialpolitische Interventionsmaßnahmen beeinflußt wird.

Vieles spricht zudem dafür, daß nicht die (wahrgenommene) Verbreitung in der gesamten Gesellschaft als Orientierungspunkt für die Bewertung der Notwendigkeit herangezogen wird. Kapteyn et al. (1978) und Kapteyn/ Wansbeck (1982:148) konnten z. B. bei der Untersuchung von Determinanten für die Höhe von subjektiv angegebenen Mindesteinkommen beobachten, daß das Mindesteinkommen umso höher angegeben wurde, je höher das Durchschnittseinkommen in der Referenzgruppe der befragten Haushalte war. Als Referenzgruppe werden in diesen Untersuchungen wie auch in den entsprechenden Vorschlägen von Muffels (1993) und Halleröd (1994) - Personen und/oder Haushalte betrachtet, die eine Reihe soziodemographischer Merkmale mit der Befragungsperson gemeinsam haben. An diesem Vorgehen scheint mir problematisch zu sein, daß damit implizit eine genauere Kenntnis der Verbreitung von Lebensstandardmerkmalen für Personen mit z. B. gleichen Bildungsabschlüssen oder ähnlichem Alter als für die Verbreitung in der Gesamtgesellschaft vorausgesetzt wird. Für die subjektive Einschätzung von Durchschnittseinkommen plausibilisiert der offensichtliche (und in öffentlichen Diskursen präsente) Zusammenhang zwischen individuell erzielbarem Einkommen und solchen Merkmalen deren Heranziehung als Orientierungshilfe. Es darf jedoch bezweifelt werden, daß ein ähnlich enger Zusammenhang zwischen soziodemographischen Merkmalen und der subjektiven Einschätzung der Verbreitung von vielfältigen Lebensstandardmerkmalen besteht. Damit soll nicht die potentielle Bedeutsamkeit von Referenzgruppen für die Verbreitungswahrnehmung bestritten werden. Allerdings müßte man nach meiner Einschätzung größere Sorgfalt bei der Definition von soziologisch relevanten Referenzgruppen verwenden und insbesondere auch die sie konstituierenden Wirkungsprinzipien benennen.

Dabei wäre unter anderm zu klären, ob sich die wahrnehmungsstrukturierenden sozialen Vergleichsprozesse immer auf ähnlichen Gruppen und/oder Personen beziehen. Gerade im Vergleich zwischen Ost- und Westdeutschland kann man demgegenüber annehmen, daß sich Personen aus Ostdeutschland am Lebensstandard der Westdeut- 
schen orientieren. Nicht zuletzt sind die nach wie vor bestehenden Unterschiede in den Lebensverhältnissen in vielen Dimensionen zwischen Ost- und Westdeutschland fast täglich Gegenstand öffentlicher Diskurse. Es wird also (u.a. durch die Massenmedien) den Ostdeutschen der Eindruck vermittelt, $\mathrm{da} ß$ sie weniger haben, als in einem großen Teil der Gesellschaft üblich ist. Kanning und Mummendey (1993) konnten z. B. zeigen, daß Personen aus Ostdeutschland die wirtschaftliche Situation als subjektiv wichtige Vergleichsdimension bewerten. Gleichzeitignehmen sie wahr, daß sie auf dieser Dimension gegenüber den Westdeutschen benachteiligt sind. Als Strategie zur Bewältigung der daraus resultierenden negativen sozialen Identität wird hierbei sehr häufig das Streben nach Assimilation genannt. Das mag dazu führen, daß viele der erhobenen Lebensstandardmerkmale von Ostdeutschen häufiger als notwendig bewertet werden.

$\mathrm{H}_{5}$ : Befragte aus den neuen Bundesländern halten die erfragten Lebensstandardmerkmale häufiger für notwendig als Befragte aus den alten Bundesländern.

Allerdings ist auf Basis der bislang vorliegenden Forschungsergebnisse kaum vorherzusagen, in welchem Ausmaß die oben erwähnten Vergleichsprozesse sich in gleicher Weise auf die Bewertung der Lebensstandarditems als ,unbedingt notwendig' auswirken. Ohne deshalb hierzu eine eigene Hypothese zu formulierten, werde ich dieser Frage unten nachgehen.

Die Aufforderung, den notwendigen Lebensstandard von allen Menschen in Deutschland zu bewerten, stellt möglicherweise eine Überforderung der Befragten dar. Die Beantwortung der Frage, ob ein bestimmtes Gut oder eine bestimmte Tätigkeit für eine Person, die möglicherweise in einer ganz anderen Lebenssituation als man selbst ist (z. B. wesentlich älter ist oder im Gegensatz zur Befragungsperson in einem Familienzusammenhang lebt), zur Erlangung eines ,normalen, ausreichend guten' Lebensstandards erforderlich ist, setzt ein erhebliches Abstraktionsvermögen voraus. $\mathrm{Ob}$ eine solche Abstraktionsleistung in einer zeitlich begrenzten Interviewsituation erbracht werden kann, darf bezweifelt werden. Es wäre deshalb kaum verwunderlich, wenn Menschen bei der Beantwortung einer solchen Frage ihre eigene Lebenssituation als Bewertungsmaßstab heranziehen. Die Frage „Was braucht man zum Leben?“ läßt sich spontan wohl am ehesten für die eigene Lebenssituation beantworten. Die nächstliegende Hypothese, die sich daraus ableiten läßt, ist ein vermutlich recht enger Zusammenhang zwischen der eigenen Verfügbarkeit von Lebensstandardmerkmalen und ihrer Bewertung:

$\mathrm{H}_{6}$ : Personen, die persönlich über ein Merkmal des notwendigen Lebensstandards verfügen, rechnen dieses auch häufiger zum notwendigen und/oder minimal notwendigen Lebensstandard als solche, die aus finanziellen oder anderen Gründen darauf verzichten (müssen).

Für diese Hypothese spricht zudem eine zu vermutende wechselseitige Beeinflussung von individueller Entscheidung für den Erwerb bzw. die Teilnahme an einem Lebensstandardmerkmal und der Bewertung der Notwendigkeit. In der Regel werden Menschen aus Gründen der Dissonanzreduktion bestrebt sein, an Items, die sie normativ für alle Menschen (oder zumindest für solche in einer ähnlichen Lebenssituation) als notwendig betrachten, auch persönlich zu partizipieren. Ebenso ist zu erwarten, daß die Neigung Items zum notwendigen Lebensstandard zu rechnen geringer ist, wenn man persönlich ohne auskommen muß oder aufgrund von Präferenzen, die von der Mehrheitsmeinung abweichen (Halleröd et al. 1997: 215) freiwillig darauf verzichtet.

Zudem hat eine Reihe der erfragten Lebensstandardmerkmale für Menschen in unterschiedlichen Lebenssituationen aus ganz praktischen Gründen eine unterschiedliche Relevanz. Das betrifft vor allem solche Merkmale, für die von unterschiedlichen Bedarfen bei verschiedenen Bevölkerungsgruppen ausgegangen werden muß (z. B. ein Telefon für ältere Alleinlebende, ein Auto für Personen aus ländlichen Gegenden). Für solche Items ist zu vermuten, daß verstärkt die eigene Lebenssituation als Bewertungsmaßstab herangezogen wird, wenn nicht explizit die Bewertung mit Referenz auf eine bestimmte Lebenssituation erfragt wird - wie z. B. bei den Merkmalen zum Lebensstandard von Kindern und den Arbeitsplatzmerkmalen. Es spricht einiges dafür anzunehmen, daß sich die Abstraktion von persönlichen Wünschen und Aspirationen für viele Merkmale auf den (antizipierten) Bedarf von Menschen und Haushalten in einer ähnlichen Lebenssituation beschränkt. Referenzgruppen haben also nicht nur als potentieller Orientierungsrahmen für die Wahrnehmung der Verbreitung von Lebensstandardmerkmalen eine Bedeutung sondern auch als Referenzrahmen für die Einschätzung von Bedarfslagen und damit für die im Interview artikulierte Notwendigkeitsbewertung.

$\mathrm{H}_{7}$ : Die Notwendigkeitsbewertung variiert für Merkmale, die für Personen und Haushalte in unterschiedlichen Lebenssituationen eine unterschiedliche (praktische) Bedeutung haben mit der eigenen Lebenssituation. 
Charakteristika des zu bewertenden Merkmals

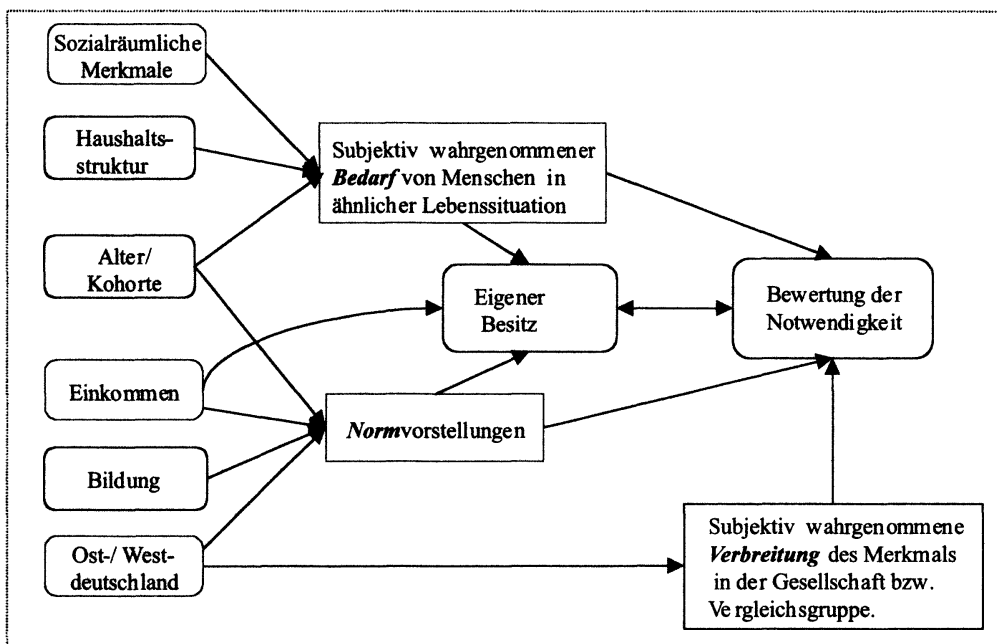

Abb. 1 Ein Analysemodell für die Bewertung der Notwendigkeit Quelle: Eigene Darstellung
Naheliegenderweise wird die Verbreitung solcher Merkmale bei Haushalten in entsprechenden Lebenssituationen auch höher sein als bei anderen. $\mathrm{Ob}$ sich also ein eigenständiger Effekt der Bedarfswahrnehmung für die ,eigene Referenzgrup$\mathrm{pe}^{\prime}$ ausmachen läßt, ist deshalb mit geeigneten multivariaten Verfahren zu prüfen. Der (wahrgenommene) Bedarf dürfte für eine Reihe von Items mit einer Haushaltstypologie (insbesondere Einvs. Mehrpersonenhaushalte jeweils mit und ohne Kindern) sowie mit dem Lebensalter variieren. Weiterhin unterscheiden sich die Bedarfslagen für eine Reihe von Items wie z. B. das Auto oder die Verfügbarkeit von Infrastruktur vermutlich auch nach einer Stadt-Land-Differenzierung.

Bei anderen Items lassen sich die im Interview geäußerten normativen Notwendigkeitsbewertungen vermutlich eher auf lebensstiltypische Werthaltungen der Befragungsperson als auf die unter Bezug auf die eigene Lebensituation antizipierten $\mathrm{Be}$ darfslagen zurückführen. Dabei ist insbesondere an solche Lebensstandardmerkmale zu denken, deren Besitz oder Ausübung als Teil der Unterscheidung zwischen unterschiedlichen Lebensstilgruppen (Müller 1992; Spellerberg 1996) angesehen wird. Für Merkmale, die zum einen nicht dem reinen Grundbedarf zuzurechnen sind und die andererseits z. B. Ausdruck von bestimmten Formen des interaktiven Verhaltens (Freizeitaktivitäten, Informationsverhalten) sind, ist davon auszugehen, da $B$ deren Bedeutung von Personen aus unterschiedlichen Lebensstilgruppen unterschiedlich bewertet wird. Gerade geteilte kulturelle Vorlie- ben und expressive Stilisierungen der Lebensweisen werden von den Lebensstilforschern als konstitutive Elemente von Lebensstilen hervorgehoben und als Ergänzung und Verfeinerung von klassischen Sozialstrukturanalysen (Müller 1992: 369) betont. Unter anderem Bildung und Alter werden dabei als wichtige Einflußgrößen für die Zuordnung von Individuen zu Lebensstilen gesehen (Spellerberg 1996: 214). Auf dieser Basis könnte man vermuten, daß z. B. höher gebildete Personen Merkmale der „häuslichen Unterhaltungskultur“ seltener zum notwendigen Lebensstandard rechnen als Personen mit niedrigen Bildungsabschlüssen. Von den erfragten Lebensstilmerkmalen wäre hier z. B. an den Farbfernseher oder den Videorecorder zu denken.

$\mathrm{H}_{\mathrm{g}}$ : Für Merkmale, die Ausdruck von bestimmten lebensstiltypischen Werthaltungen oder Verhaltensweisen sind, ist mit einer Abhängigkeit der Notwendigkeitsbewertung von Bildungsniveau, Alter und Einkommen zu rechnen.

Abbildung 1 faßt die durch die obigen Hypothesen umrissene Analysestrategie für mögliche Bewertungsunterschiede zusammen.

Die zu erwartenden Unterschiede in der Bewertung der Notwendigkeit lassen sich nicht für alle erfragten Lebensstandarddimensionen auf die gleichen Hypothesen und Theoriestränge zurüickführen. Diese Abhängigkeit der zu erwartenden Einflüsse von den unterschiedlichen Valenzen der Merkmale ist in Abbildung 1 durch den gestrichelten Rahmen um das Analysemodell symbolisiert. Die oben formulierten Hypothesen stellen im wesentlichen auf drei Einflußgrößen für die Bewer- 
tung der Notwendigkeit ab, deren relative Bedeutung wiederum von den spezifischen Charakteristika des zu bewertenden Merkmales abhängen dürfte: 1 . die subjektiv wahrgenommene Verbreitung von Merkmalen hat für alle Merkmale einen mehr oder weniger deutlichen Einfluß. 2. hängt die Bewertung der Notwendigkeit mit verschiedenen Indikatoren für den subjektiv wahrgenommenen Bedarf von Menschen in ähnlichen Lebenssituationen zusammen und 3. unterscheiden sich die Bewertungen nach verschiedenen Indikatoren für die Zugehörigkeit zu unterschiedlichen Lebensstilgruppen. Nicht zuletzt ist auch von einem recht engen wechselseitigen Zusammenhang zwischen der normativen Bewertung der Notwendigkeit von Lebensstandardmerkmalen für alle Menschen und der individuellen Verfügung über diese auszugehen. Im allgemeinen ist zu erwarten, daß sich die hier umrissenen Einflüsse sowohl für die Bewertungen als ,notwendiger Lebensstandard' als auch als ,minimal notwendiger Lebensstandard ' zeigen.

\section{Ergebnisse}

Mit $\mathbf{H}_{1}$ bis $\mathbf{H}_{4}$ wurden Hypothesen über die Bewertung der Notwendigkeit formuliert, die sich auf Anteile in der gesamten Population beziehen. Betrachten wir deshalb zunächst die Bewertung der Notwendigkeit für alle erfragten Merkmale im Durchschnitt über alle befragten Personen. Tabelle 1 zeigt neben der Notwendigkeitsbewertung (Spalten A und B) in Spalte C auch den Anteil von Befragten, die über das jeweilige Merkmal verfügen. Die Items sind in der Tabelle nach der anteiligen Notwendigkeitsbewertung (Spalte A) geordnet. Die Sortierung erfolgte zur besseren Übersicht über die erfragten Lebensstandarddimensionen getrennt nach den insgesamt 27 Items des allgemeinen Lebensstandards, den jeweils ergänzenden Items für den Lebensstandard von Erwerbstätigen und Arbeitssuchenden sowie von Familien mit Kindern und schließlich den Infrastrukturmerkmalen.

Im Sinne von Hypothese 1 bezeichne ich im folgenden Items, die von mehr als $90 \%$ der Bevölkerung zum notwendigen Lebensstandard gerechnet werden (Spalte A), als Grundbedarf. Nach dieser Abgrenzung sind 11 Merkmale des allgemeinen Lebensstandards aus allen erfragten Lebensstandarddimensionen mit Ausnahme der Sozialkontakte, alle vier Arbeitsplatzmerkmale sowie die Verfügbarkeit von öffentlichem Nahverkehr in der Nähe der Wohnung zum Grundbedarf zu rechnen.
Für diese Merkmale ist nicht mit erheblich abweichenden Bewertungen durch größere Teile der Bevölkerung zu rechnen. $\mathrm{Ob}$ sich dennoch für einzelne dieser Merkmale nennenswerte Unterschiede nach den vermuteten Einflüssen von wahrgenommenen Bedarfsunterschieden bzw. unterschiedlichen Normvorstellungen ausmachen lassen wird unten geprüft. Bis dahin darf man für diese Merkmale wohl von einem weitgehenden gesellschaftlichen Konsens über deren Bedeutung für einen normalen, ausreichend guten Lebensstandard ausgehen. Das gilt in dieser Deutlichkeit jedoch nur, wenn dabei auf die weitere Abgrenzung als notwendiger Lebensstandard abgestellt wird. Die aus Sicht der Befragten hohe Bedeutung dieser Items zeigt sich aber auch an der im Vergleich zu anderen Items geringen Differenz zwischen den Prozentwerten in den Spalten A und B. Nur diese Items sowie ein Telefon und die Verfügbarkeit eines Arztes in der Nähe werden auch von mehr als der Hälfte der Bevölkerung als ,unbedingt notwendig' bewertet. Bei fast allen Grundbedarfsmerkmalen äußerten sich sogar mehr als zwei Drittel der Befragten in diesem Sinn.

Erweitert man die Betrachtung auf solche Items, die von einer Mehrheit der Befragten zum notwendigen Lebensstandard gerechnet werden, so ist deutlich zu erkennen, daß nicht nur Dinge des (materiellen) Grundbedarfes hierzu zählen. Auch Merkmale, die eher auf die Lebensqualität und die Teilhabe an sozialen Aktivitäten zielen, werden von einer großen Mehrheit der Bevölkerung als (eher) notwendiger Bestandteil des ,normalen, ausreichend guten' Lebensstandards gesehen. Lediglich fünf der insgesamt 42 Items werden von weniger als der Hälfte der Population als notwendig betrachtet. Mack und Lansley (1985: 56ff) werten ein vergleichbares Ergebnis ihrer Befragung als Argument dafür, daß Armut - in ihrer Lesart definiert als erzwungener Verzicht auf ,socially perceived necessities ${ }^{6}$ - aus der Sicht einer deutlichen Bevölkerungsmehrheit nicht erst beginnt, wenn Merkmale des Grundbedarfs fehlen. Hierzu ist jedoch einschränkend zu bemerken, daß Mack und Lansley (wie auch alle anderen einschlägigen Autoren) die Bewertung der Notwendigkeit von vornherein mit einer dichotomen Antwortvorgabe erfragt hatten. Wenn man den Befragten eine Abstufung der Notwendigkeitsbewertung ermöglicht und als Abgrenzungskriterium für den minimal notwendigen Lebensstandard ausschließlich die Nennung ,unbedingt notwendig' heranzieht (Spalte B), so ergibt sich im Kontrast zu Mack und Lansleys Ergebnis die Schlußfolgerung, daß sich 
Tabelle 1 Notwendigkeitsbewertungen für die erfragten Aspekte des Lebensstandards.

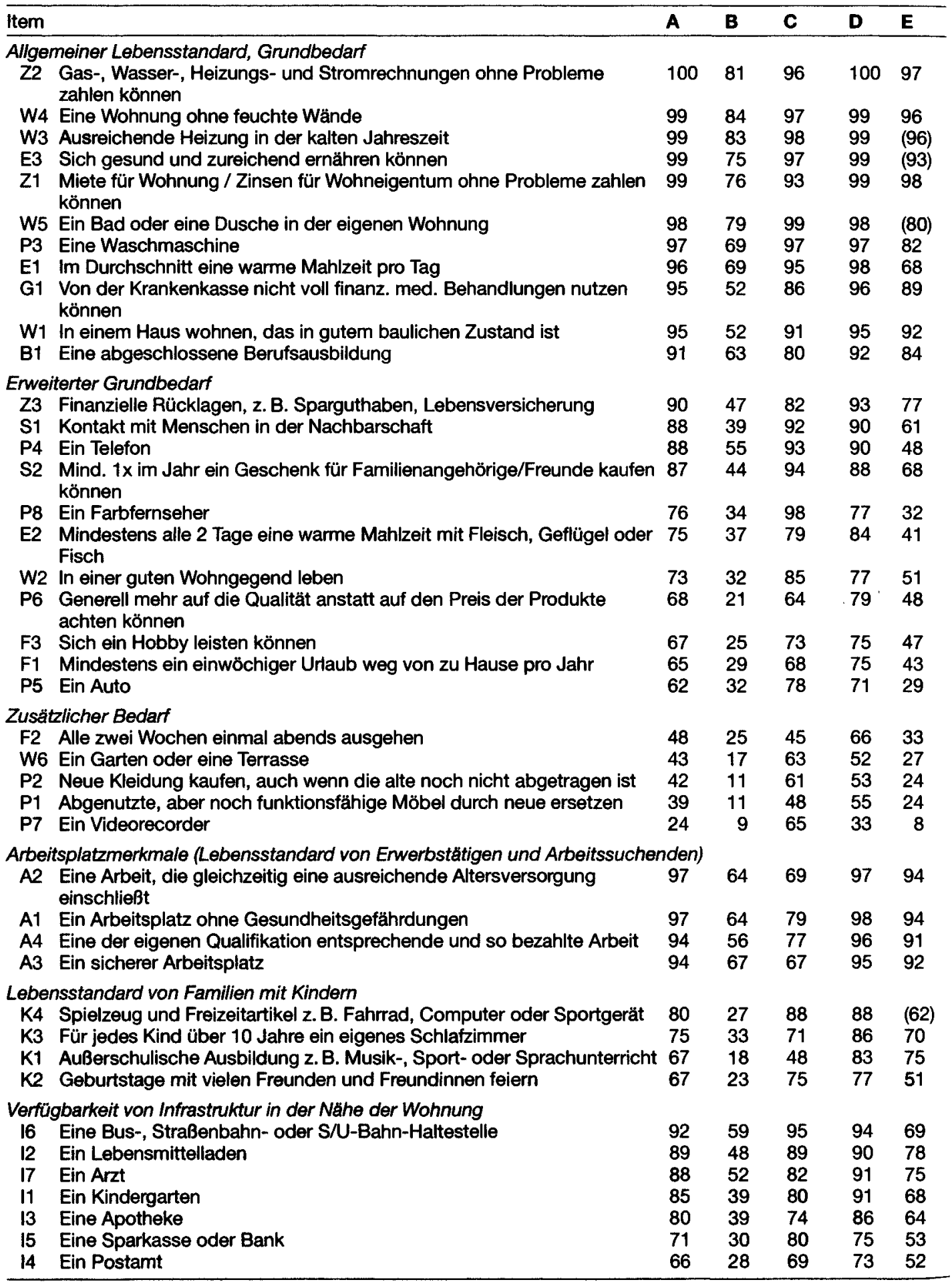


Anmerkungen zu Tabelle 1: Spalten A - C: Prozentualer Anteil aller Befragten, die das Item als "eher notwendig oder unbedingt notwendig“ (A) bzw. „unbedingt notwendig" (B) bewerten. In Spalte C der prozentuale Anteil von Befragten, die über das Item verfügen. Hiebei ist zu beachten, daß die Frage nach der Verfügbarkeit bei den Items zu Arbeitsplatzmerkmalen nur Erwerbstätigen vorgelegt wurde $(n=1469)$ und bei den Items zum Lebensstandard von Kindern nur Personen aus Haushalten, in denen Kinder unter 18 Jahren leben $(n=816)$. Spalten D und E: Prozentualer Anteil der Nennung "Eher notwendig oder unbedingt notwendig" für Personen, die persönlich über das jeweilige Item verfügen (D) und der entsprechende Anteil für Personen, die nicht über das Item verfügen (Spalte E). Prozentwerte in Klammern ( ) wenn die Berechnung auf weniger als 100 Fällen basiert.

Quelle: Sozialwissenschaftenbus III/96, gewichtete Ergebnisse (Fallzahlen ungewichtet)

auf diesem minimalen Niveau nur für die oben als Grundbedarf bezeichneten Merkmale eine Mehrheit findet. Inwieweit daraus der Schluß zu ziehen ist, daß lediglich diese Merkmale als Armutsindikatoren geeignet sind ist erneut nur normativ zu entscheiden.

Vergleicht man die Prozentwerte aus den Spalten $A$ und $C$ von Tabelle 1 , so scheinen die für viele Items recht ähnlichen Werte den in $\mathrm{H}_{2}$ vermuteten engen Zusammenhang zwischen Verbreitung und Bewertung der Notwendigkeit zu bestätigen. Lebensstandardmerkmale, über die ein großer Teil der Bevölkerung verfügt, werden auch zu recht ähnlichen Anteilen zum notwendigen Lebensstandard gerechnet. Das gilt nicht nur für die Grundbedarfsmerkmale sondern auch für die meisten Items, die weniger einheitlich zum notwendigen Lebensstandard gezählt werden. Die Korrelation der Prozentwerte in Spalte A mit dem prozentualen Anteil der Verfügbarkeit in Spalte $C$ beträgt 0,74 ; die Korrelation für die ausschließliche Nennung ,unbedingt notwendig' (Spalte B) ist mit 0,71 nur geringfügig niedriger.

Bei genauerer Betrachtung finden sich jedoch sowohl Items, für die sich das in $\mathrm{H}_{3}$ vermutete Zurückbleiben der Verbreitung hinter dem Anteil derer, die diese dem notwendigen Lebensstandard zurechnen, beobachten läßt, als auch solche, die im Einklang mit $\mathbf{H}_{4}$ zwar verbreitet sind, jedoch im Vergleich dazu eher selten als notwendig bewertet werden. Für die Gültigkeit von $\mathrm{H}_{3}$ sprechen am deutlichsten die Unterschiede bei den Arbeitsplatzmerkmalen. Gegenüber mehr als $90 \%$ der Befragten, die diese vier Items zum notwendigen Lebens- standard rechnen, verfügen ,nur 70 bis maximal 80 Prozent der befragten Erwerbstätigen ${ }^{4}$ über entsprechende Arbeitsplätze. Weitere Merkmale, die für den empirischen Gehalt von $\mathrm{H}_{3}$ sprechen, sind wenn auch mit nicht ganz so großen Diskrepanzen - die abgeschlossene Berufsausbildung (B1), die Möglichkeit, Kindern eine außerschulische Ausbildung zu ermöglichen (K1), das Gesundheitsitem (G1) sowie die Verfügbarkeit finanzieller Rücklagen (Z3). Bei diesen Merkmalen handelt es sich durchweg um solche Bereiche des alltäglichen Lebens, die den (kurzfristigen) Einflußmöglichkeiten des Individuums weitgehend entzogen sind.

Insgesamt sechs Items sind zwar für eine Mehrheit der Befragten verfügbar, werden aber von einem um mindestens 10 Prozentpunkte niedrigeren Teil der Befragten zum notwendigen Lebensstandard gerechnet. Das betrifft ausschließlich Items aus den Bereichen des materiellen persönlichen Bedarfes und der Verfügbarkeit von Wohnraum mit bestimmten Eigenschaften. Am deutlichsten ist dieser Unterschied bei der Bewertung des Videorecorders: Fast zwei Drittel der Befragten verfügen über einen solchen, doch nur ein knappes Viertel rechnet ihn zum notwendigen Lebensstandard. Ähnliches gilt für den Farbfernseher (P8), das Leben in einer guten Wohngegend (W2), ein Auto (P5), Garten oder Terrasse (W6) und die Anschaffung neuer Kleidung (P2). Bei diesen Merkmalen ist weiterhin auffällig, daß sie trotz ihrer vergleichsweise großen Verbreitung nur von maximal einem Drittel der Befragten zum minimal notwendigen Lebensstandard (Spalte B) gezählt werden.

\subsection{Individuelle Verfügbarkeit und Notwendigkeitsbewertung}

In den Spalten D und E von Tabelle 1 findet sich der prozentuale Anteil von Befragten, die das jeweilige Merkmal als eher notwendig bewerten, differenziert nach der persönlichen Verfügbarkeit dieses Items. Zunächst ist festzuhalten, daß Personen, die persönlich über ein Item verfügen (Spalte D), dieses auch durchgängig öfter für notwendig zur Erreichung oder Sicherstellung eines normalen, ausreichend guten Lebensstandards halten als Personen, die nicht über das Item verfügen (Spalte E). Das Ausmaß dieser Bewertungsunterschiede variiert allerdings erheblich mit dem jeweiligen Item. Für die Merkmale des Grundbedarfs zeigen sich in der Regel nur marginale Unterschiede.

\footnotetext{
${ }^{4} \mathrm{Zu}$ der Einschränkung auf Erwerbstätige bei diesen Items siehe die Anmerkung zu Tabelle 1.
} 


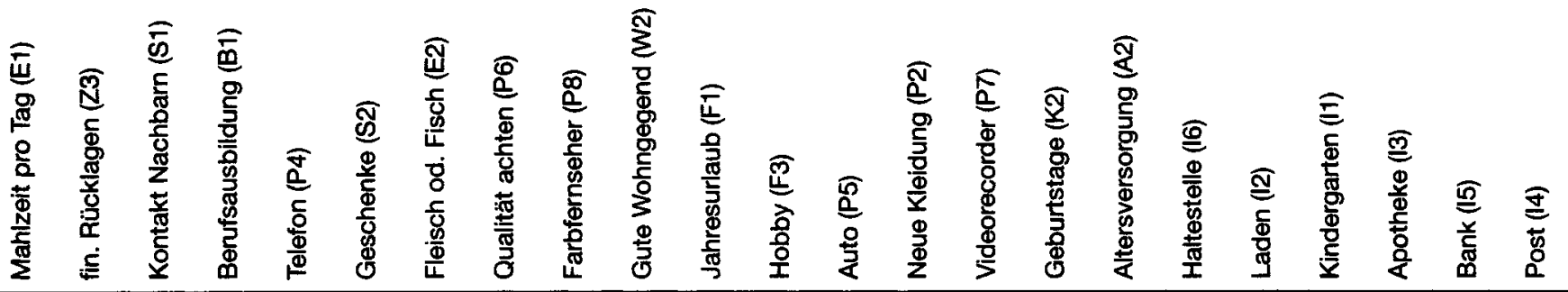

Referenz (a)

$\begin{array}{lllllllllllllllllllllllll}\text { \% Notwendig } & 98 & 94 & 90 & 89 & 87 & 86 & 82 & 77 & 75 & 75 & 73 & 72 & 68 & 54 & 33 & 70 & 98 & 96 & 92 & 89 & 88 & 79 & 78\end{array}$

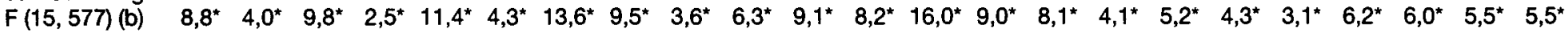

\section{Region (Westdeutschland)}

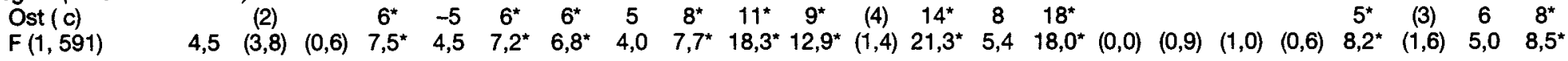

\section{Wohnort (Großstadt)}

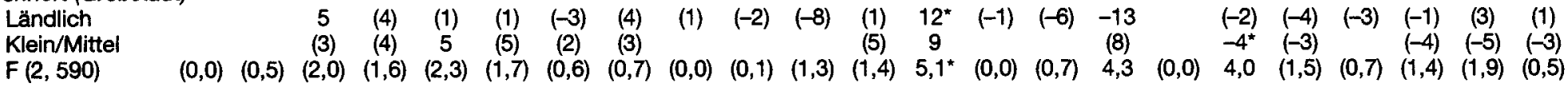

$F(2,590)$

$(0,0)$

Haushaltstyp (Paar $<65)$
Allein $>=65$

(5)

Raar $>=65$

Paar m. Kindern

(2) (3) (3)

Alleinerziehend

Sonstige

$F(6,586)$

(4) (5) (5)

$\begin{array}{ccccc}(7) & & & (6) & (7) \\ (-9) & (2) & (7) & (-8) & (-9)\end{array}$

(5) (5) (5)

$\begin{array}{lllllll}(-6) & -12 & -21^{*} & (-8) & (-9) & - & -\end{array}$

(3)

(3) (3)

Alter (30-49)

\begin{tabular}{|c|c|c|c|c|c|c|c|c|c|c|c|c|c|c|c|c|c|c|c|c|c|c|c|}
\hline $\begin{array}{l}18-29 \\
50-64 \\
F(2,590)\end{array}$ & $(2,5)$ & $(0,5)$ & $\begin{array}{l}(-4) \\
(2) \\
3,7\end{array}$ & $(0,2)$ & $(0,1)$ & (3) & $(0,0)$ & $(0,1)$ & $\begin{array}{c}(3) \\
(4) \\
(0,7)\end{array}$ & $\begin{array}{c}-7 \\
(0,1)\end{array}$ & $(0,2)$ & $(2,5)$ & $\begin{array}{c}(4) \\
-9 \\
4,8^{*}\end{array}$ & $\begin{array}{c}-9 \\
(-7) \\
3,0\end{array}$ & $\begin{array}{c}(7) \\
(-7) \\
4,0\end{array}$ & $\begin{array}{c}(3) \\
(8) \\
(0,3)\end{array}$ & $(2,1)$ & $(0,2)$ & -4 & $(0,4)$ & $(-4)$ & $\begin{array}{l}(-2) \\
(0,5)\end{array}$ & $\begin{array}{l}-8^{*} \\
4,0\end{array}$ \\
\hline
\end{tabular}

(Fortsetzung nächste Seite) 


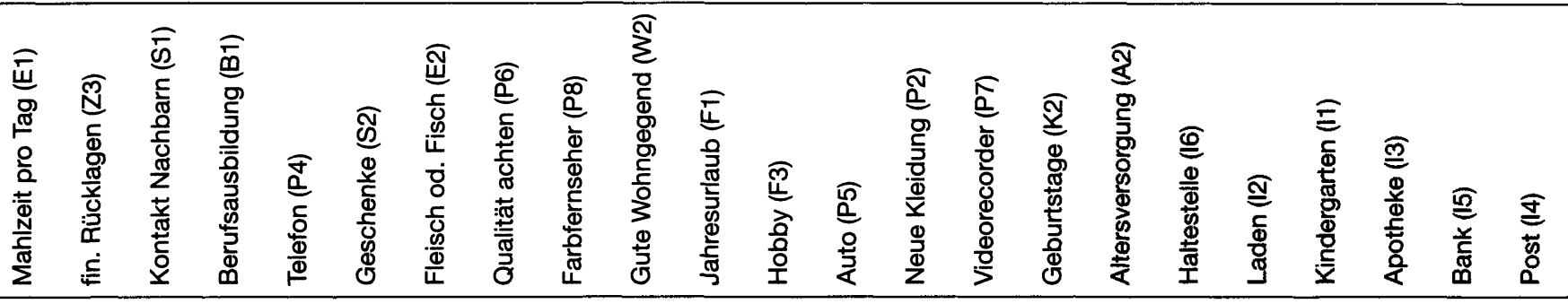

Aquivalenzeinkommen (2.066 DM)

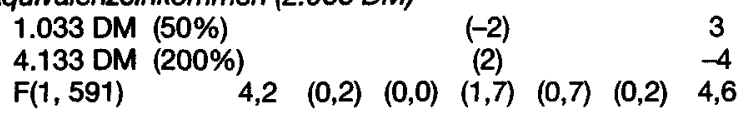

\section{3}

$\begin{array}{llll}(-4) & (-2) & -5 & (-3)\end{array}$

$F(1,591)$

$4,2 \quad(0,2$

$(0,0)(1,7)$

$-4$

$(0,5)(0,0)$

(4) (3) $6 \quad$ (3)

(4)

(-6) $-3^{*}$

(3) (2)

SchulabschluB (Hauptschule/kein AbschluB)
Mittlere Reife
Abi/Poly/FHS
(3) (4)
$\begin{array}{llll} & & \text { (3) } \\ -12^{*} & -8 & \text { (3) }\end{array}$
(2)
$\begin{array}{llll}-8 & (-3) & -9 & (11)\end{array}$
$-3$
$F(2,590)$
(1,3) $\quad 4,6$
$\begin{array}{lll}(0,3) & (2,5) \quad(2,5) \quad(1,1)\end{array}$
$(0,9) \quad(0,1) \quad 5,8^{*} \quad 3,6$
$(0,6) \quad(0$,
$\begin{array}{ccccc} & -8 & (-3) & -9 & (11) \\ (0,2) & 4,0 & (0,6) & 3,5 & (2,9)\end{array}$
$\begin{array}{lll}(0,1) & 3,4 & (-3) \\ (1,3)\end{array}$

$\begin{array}{llll}(1,4) & (0,0) & (3,0) & (2,5)\end{array}$

Verfügbarkeit ('Ja, verfügbar/wird ausgeübt')

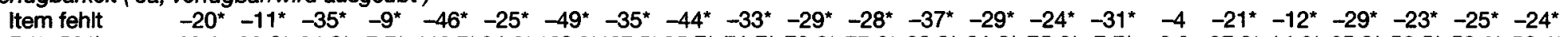

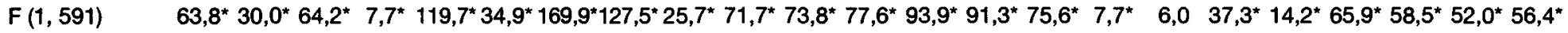

Anmerkungen : (a) Geschätzter prozentualer Anteil der Nennung ,Notwendig' für eine Person, bei der alle Merkmale die Referenzausprägung (bei den Merkmalen in Klammern genannt) aufweisen. (b) Teststatistik eines angepaßten Wald-Tests für den Einfluß aller Merkmale (Referenz) bzw. aller Kategorien der unabhängigen Variablen. (c) Geschätzte Abweichung von der Referenz in Prozentpunkten. Ref.: Referenzkategorie bei dem ,Kinderitem': Geburtstage mit vielen Freunden feiern (K2). - nicht geschätzt da Haushalts-Typ hier nicht einschlägig. Signifikanzen der Logit-Koeffizienten und der Wald-Tests: * $\alpha=0,01$, ohne Stern $\alpha=0,05$, in Klammern () nicht signifikant.

Quelle: Sozialwissenschaftenbus III/96, gewichtete Ergebnisse. 
Demgegenüber wird z.B. das zweiwöchentliche abendliche Ausgehen (F2) von zwei Dritteln der Befragten, die sich das erlauben (können) auch als Bestandteil des notwendigen Lebensstandards betrachtet, während Personen die darauf verzichten (müssen) lediglich zu einem Drittel diese Auffassung vertreten.

Die Abhängigkeit der Notwendigkeitsbewertung von den in Abbildung 1 skizzierten unabhängigen Variablen wurde für jedes der erfragten Items durch eine logistische Regression modelliert. Abhängige Variable dieser Modelle ist die am weiteren Kriterium (Tabelle 1, Spalte A) dichotomisierte Notwendigkeitsbewertung. Aus Platzgründen wird auf eine getrennte Darstellung entsprechender Schätzergebnisse für die Bewertungsunterschiede bei einer Operationalisierung der abhängigen Variablen am engeren Kriterium nach Spalte $B$ verzichtet. Auffällige Unterschiede werden im folgenden jedoch kommentiert. Mit Ausnahme des Äquivalenzeinkommens nach der neueren OECD-Skala ${ }^{5}$ - das in logarithmierter Form als kontinuierliche unabhängige Variable berücksichtigt wurde - sind alle anderen erklärenden Variablen durch eine geeignete Dummy-Kodierung aufgenommen worden. Die Haushaltstypologie umfaßt dabei auch eine Differenzierung zwischen Personen unter und über 65 Jahren. In der eigenständigen Altersklassierung fehlt deshalb die Altersklasse der 65 jährigen und älteren. Die Schätzergebnisse sind in Form der durch das Modell vorhergesagten Abweichung von der Bewertung durch eine (theoretische) Referenzperson ausgewiesen. Dieser Vergleichswert ist in der ersten Zeile von Tabelle 2 ausgewiesen und entspricht dem vorhergesagten prozentualen Anteil der Bewertung als ,eher notwendig' für Personen, die gleichzeitig bei allen unabhängigen Variablen die Referenzausprägung ${ }^{6}$ aufweisen. Diese Form der Dar-

\footnotetext{
${ }^{5}$ Mit dieser Äquivalenzskala wird das Ziel verfolgt, die Einkommensposition von Haushalten unterschiedlicher Größe und Zusammensetzung vergleichbar zu machen. Für die hier verwendete Skala wird hierzu für die erste Erwachsene Person im Haushalt ein Gewicht von 1, für weitere Personen über 15 ein Gewicht von 0,5 und für Kinder unter 15 von 0,3 verwendet.

${ }^{6}$ Die Kodierung der Dummy-Variablen wurde so gewăhlt, daB die Referenzkategorie der jeweils am häufigsten besetzten Kategorie der unabhängigen Variablen entspricht. Diese ist in der Tabelle jeweils hinter der Variablenbezeichnung ausgewiesen. Konkret bezieht sich die erste Zeile der Tabelle also auf die durch das Modell geschätzte Bewertung durch westdeutsche, in einer Großstadt lebende Personen aus Paarhaushalten ohne Kinder
}

stellung hat gegenüber den geschätzten Logit-Koeffizienten den Vorzug, daß die Größenordnung der zu erwartenden Bewertungsunterschiede besser veranschaulicht wird.

Für jede der unabhängigen Variablen ist neben der für die einzelnen Ausprägungen multivariat geschätzten Abweichungen von der Referenz in Prozentpunkten die Teststatistik für einen angepaßten Wald-Test ${ }^{\top}$ ausgewiesen. Dieser Test dient zur Prüfung der Nullhypothese, daß von der jeweiligen Variablen kein statistisch signifikanter Einfluß auf die Notwendigkeitsbewertung ausgeht. Die entsprechende Teststatistik für die Referenz ist ein Test für den simultanen Einfluß aller im Modell berücksichtigten Variablen. Tabelle 2 zeigt nur Ergebnisse für solche Items, bei denen überhaupt signifikante Unterschiede in der Notwendigkeitsbewertung feststellbar sind. Da sich mit Ausnahme einiger Grundbedarfsmerkmale für alle Items signifikante Unterschiede nach der persönlichen Verfügbarkeit zeigten, wurden auch solche Items nicht berücksichtigt, für die nicht mindestens für eine weitere Variable signifikante Unterschiede beobachtbar waren. Vorhergesagte Unterschiede, die weniger als 2 Prozentpunkte betragen, sind ebenfalls nicht in der Tabelle dargestellt.

Betrachtet man zunächst die Schätzergebnisse für die Ost-West-Differenzierung, so bestätigt sich für insgesamt 13 Lebensstandardmerkmale die Hypothese $\left(\mathrm{H}_{5}\right)$, daß Ostdeutsche öfter von der Notwendigkeit von Lebensstandardmerkmalen überzeugt sind. Lediglich das Telefon wird von ihnen seltener als von der (rechnerischen, westdeut-

im Alter zwischen 30 und 49 Jahren mit einem Äquivalenzeinkommen von $2.066 \mathrm{DM}$ (dem Stichprobendurchschnitt) und Hauptschulabschluß, die über das jeweilige Item verfügen. Für die Arbeitsplatzmerkmale wurden Personen über 65 Jahren aus der Analyse ausgeschlossen. Für die Items zum Lebensstandard von Kindern reduziert sich die analysierbare HH-Typologie auf 3 Typen. Das hat u.a. zur Folge, daß die Referenzkategorie für diese Items der Paarhaushalt mit Kindern ist.

7 Es handelt sich hier um angepaßte Wald-Tests (Korn/ Graubard 1990; Stata Corporation 1997), weil die zugrundeliegende Stichprobe keine einfache Zufallsstichprobe ist, sondern disproportional geschichtet wurde (Überrepräsentation Ostdeutscher) und durch das ADM-Design Klumpungseffekte aufweist. Die Schätzungen und Tests wurden deshalb mit den Prozeduren svylogit und svytest von STATA 5.0 durchgeführt. Die Teststatistiken sind nach diesem Verfahren nicht $\mathrm{CHI}^{2}$ verteilt sondern folgen näherungsweise einer F-Verteilung. Die anzusetzenden (Nenner-)Freiheitsgrade beziehen sich auf die Anzahl der gezogenen primären Stichprobeneinheiten (Kalton 1983). 
schen) Referenzperson als notwendig betrachtet. Das ist insofern bemerkenswert, als dieses Ergebnis unter Kontrolle der individuellen Verfügbarkeit zustande kommt. Der beobachtete Unterschiede ist deshalb nicht als individuelles Streben nach persönlich fehlenden Merkmalen zu interpretieren, sondern als deutlicher Hinweis auf zwischen den beiden Landesteilen unterschiedliche normative Bewertungen. Die oben dargestellten möglichen Erklärungen für derartige Unterschiede sollen hier nicht wiederholt werden. Obwohl sich für keine der anderen erklärenden Variablen (mit Ausnahme der individuellen Verfügbarkeit) bei mehr Items signifikante Bewertungsunterschiede feststellen lassen, kann nicht übersehen werden, da $B$ die vorhergesagten Unterschiede substantiell nicht besonders groß sind. Lediglich bei den Items Auto (P5), Videorecorder (P7) und gute Wohngegend (W2) betragen die vorhergesagten Unterschiede mehr als 10 Prozentpunkte. Die Bewertungen des notwendigen Lebensstandards fallen zwischen den beiden Landesteilen deshalb auch nicht völlig auseinander.

Wenig nennenswerte Unterschiede zeigen sich nach der Differenzierung nach BIK-Gemeindegrößeklassen. Erwartungsgemäß wird ein Auto von Befragten aus ländlichen Gebieten (BIK Gemeindegrößenklasse $\leq 5.000$ ) um 12 Prozentpunkte und von Personen aus Klein- und Mittelstädten um 9 Prozentpunkte häufiger zum notwendigen Lebensstandard gerechnet als von Großstädtern (BIK $\geq 100.000$ ). Auffällig ist noch, daß Personen aus ländlichen Gebieten es um 13 Prozentpunkte seltener als Stadtbewohner zum notwendigen Lebensstandard von Familien mit Kindern rechnen, Kindergeburtstage mit vielen Freunden feiern zu können (K2). Alle anderen Unterschiede sind entweder statistisch nicht signifikant oder substantiell eher klein. Wiederum im Vergleich zu Stadtbewohnern wird der Kontakt mit der Nachbarschaft (S1) von ländlich lebenden Menschen um 5 Prozentpunkte häufiger zum notwendigen Lebensstandard gerechnet. Personen aus Klein- und Mittelstädten zählen das Telefon (P4) mit einer Differenz von 5 Prozentpunkten etwas häufiger und die Verfügbarkeit einer Haltestelle in der Nähe der Wohnung (I6) um 4 Prozentpunkte seltener hierzu.

Ähnlich gering ist der Einfluß der Haushaltstypologie. Betrachtet man zunächst nur die Teststatistiken für den Einfluß der Haushaltstypologie insgesamt, so läßt sich mit einer Irrtumswahrscheinlichkeit von $5 \%$ nur für drei Items die Nullhypothese zurückweisen, daß von keiner Kategorie der Typo- logie ein von Null verschiedener Effekt ausgeht. Mit Ausnahme des Autos (P5) und des Hobbys (F3), die beide von alleinlebenden älteren Personen deutlich seltener zum notwendigen Lebensstandard gerechnet werden, ist der vorhergesagte Unterschied zur Referenzperson für keinen der wenigen signifikanten Effekte größer als 10 Prozentpunkte. Ebenso zeigen sich weder für eine $\mathrm{Al}$ tersdifferenzierung der unter 65jährigen, noch nach dem Äquivalenzeinkommen nennenswerte Unterschiede. Weder für Personen mit einem doppelt so hohen Äquivalenzeinkommen, noch für solche mit einem Einkommen in der Höhe der verbreiteten Einkommensarmutsgrenze von $50 \%$ des Durchschnittseinkommens zeigen sich signifikante Abweichungen von Durchschnittsverdienern in nennenswerter Höhe.

Statistisch signifikante Unterschiede, die auch substantiell eine gewisse Bedeutung haben, lassen sich neben den beobachteten Ost-West-Unterschieden bei einigen Items für Personen mit unterschiedlichen Bildungsabschlüssen feststellen. Insbesondere materielle Konsumgüter wie der Fernseher (P8), der Videorecorder (P7) und das Auto (P5) werden von höher gebildeten Personen seltener zum notwendigen Lebensstandard gerechnet. Dieses Ergebnis steht zwar im Einklang mit der vermuteten Lebensstilabhängigkeit von kulturellen Präferenzen, es kann jedoch ebenso wie bei den Ost-West-Unterschieden nicht übersehen werden, $\mathrm{da} B$ keiner der vorhergesagten Unterschiede mehr als 12 Prozentpunkte beträgt.

Wie bereits aufgrund der bivariaten Betrachtung der Bewertungsunterschiede nach der persönlichen Verfügbarkeit in Tabelle $1 \mathrm{zu}$ erwarten war, geht von dieser Variablen der mit Abstand deutlichste Einfluß auf die Notwendigkeitsbewertung für die in Tabelle 2 vertretenen Merkmale aus. Da in Tabelle 2 nur solche Items dargestellt sind, für die es nennenswerte (signifikante) Bewertungsunterschiede gibt, ist mit Ausnahme des Arbeitsplatzmerkmals A2 und der abgeschlossenen Berufsausbildung (B1) keines der oben als Grundbedarf bezeichneten Merkmale vertreten. Für diese Items zeigt sich auch der substantiell mit deutlichem Abstand geringste Einfluß der individuellen Verfügbarkeit. Bei vielen der anderen Lebensstandardmerkmale unterscheiden sich die vorhergesagten Bewertungsunterschiede demgegenüber in beträchtlicher Höhe.

Operationalisiert man die abhängige Variable für die oben dargestellten Analysen mit ausschließlichem Bezug auf den minimal notwendigen Le- 
bensstandard, so zeigen sich auch für einige der Grundbedarfsmerkmale signifikante Unterschiede in den vorhergesagten Anteilswerten. Auffällig ist dabei insbesondere, daß Personen aus den neuen Bundesländern diese durchgängig seltener als unbedingt notwendig betrachten. Das betrifft vor allem die Wohnungsmerkmale Bad oder Dusche (W5), ausreichende Heizung (W3) sowie eine Wohnung ohne feuchte Wände (W4), aber auch die beiden Ernährungsitems gesund ernähren (E3) und eine warme Mahlzeit pro Tag (E1). Mag für erstere als Erklärung der hohe Anteil von noch nicht vollständig sanierten Altbauwohnungen in den neuen Bundesländern plausibel erscheinen, so fällt eine schlüssige Erklärung für letztere Beobachtung schwer. Alle anderen feststellbaren $\mathrm{Be}$ wertungsunterschiede unterscheiden sich weder in Richtung noch Größenordnung von den oben berichteten Ergebnissen für die weitere Operationalisierung der Notwendigkeitsbewertung.

In der Zusammenschau muß man feststellen, daß sich nach den hier untersuchten Unterscheidungsmerkmalen kaum Unterschiede hinsichtlich der Bewertung des notwendigen Lebensstandards feststellen lassen. Unter Kontrolle der persönlichen Verfügbarkeit zeigen sich nur sehr wenig statistisch signifikante und substantiell bedeutsame Bewertungsunterschiede. Wo sich solche Unterschiede zeigen, sind diese konsistent mit den oben formulierten Hypothesen und betreffen in erster Linie Items des erweiterten Grundbedarfs. Dieses Ergebnis deckt sich mit den Beobachtungen von Nolan und Whelan (1996:79f) für Irland. Auch van den Bosch (1998: 146ff) berichtet entsprechende multivariate Ergebnisse für Belgien. Während sich das grundsătzliche Ergebnis einer wenig differenzierten Notwendigkeitsbewertung auch hier findet, zeigen sich dort etwas häufiger statistisch signifikante Ergebnisse.

\subsection{Empirische Konsequenzen fir die Kombination von Deprivationsitems}

Soll das Ausmaß der Teilhabe am für eine Gesellschaft als Minimum definierten Lebensstandard mit einem Maß gemessen werden, so stellt sich die Aufgabe, Informationen uber die Verfügbarkeit einzelner Lebensstandardmerkmale $\mathrm{zu}$ einem Index zu kombinieren. Die einfachste Möglichkeit um dieses zu bewerkstelligen, ist die Berechnung eines additiven Index, der die Anzahl der (aus finanziellen Gründen) fehlenden Items zählt. Dieses anschauliche Vorgehen wurde sowohl von
Townsend (1979), ${ }^{8}$ als auch von Mack und Lansley (1985) gewählt. Für Deutschland berichten AndreB/Lipsmeier (1995) Forschungsergebnisse mit einem derartigen Index. Mit unterschiedlichen theoretischen Begründungen wurde in vielen jüngeren Arbeiten für die Verwendung verschiedener Varianten von Gewichtungsfaktoren bei der Berechnung von Deprivationsindizes argumentiert. Eine der mit einer unterschiedlichen Gewichtung verbundenen Intentionen ist der aus Sicht der jeweiligen Autoren unterschiedliche Beitrag von einzelnen Items zum Ausmaß der Deprivation in Abhängigkeit von ihrer Verbreitung (Desai/Shah 1988), der durchschnittlichen Notwendigkeitsbewertung (Halleröd 1995) oder einer Kombination aus Verbreitung und Notwendigkeitsbewertung (Muffels 1993). Eine zweite Intention liegt in der durch geeignete Bestimmung der Gewichte möglichen Anpassung der Berechnungsweise an unterschiedliche Bedingungen in verschiedenen gesellschaftlichen Teilgruppen. So könnte man z. B. das Fehlen eines Autos für Personen aus Ostdeutschland mit einem höheren Gewicht in einem Deprivationsindex berücksichtigen, weil dieses von Ostdeutschen häufiger zum notwendigen Lebensstandard gerechnet wird. Einen solchen Vorschlag macht Halleröd (1995). Ebenso kann man argumentieren (Muffels 1993), daß der Beitrag von Items zum subjektiven Gefühl der Deprivation von der Verbreitung des Merkmals in der Referenzgruppe des Individuums abhängt.

Neben den oben bereits angesprochenen Problemen bei der Definition von soziologisch relevanten Referenzgruppen, ist es nicht zuletzt eine empirische Frage, inwieweit unterschiedliche Implementierungen von Deprivationsindizes auch $\mathrm{zu}$ unterschiedlichen Meßergebnissen führen. Aufgrund des oben beschriebenen vergleichsweise engen Zusammenhanges zwischen Verbreitung und anteiliger Notwendigkeitsbewertung könnte man z. B. vermuten, daß es empirisch keinen großen Unterschied macht ob bei der Berechnung von Deprivationsindizes mit ersterer oder letzterer gewichtet wird. Ebenso könnte man aufgrund der zumindest unter Verwendung multivariater Me-

${ }^{8}$ Townsend zählt allerdings die einfache Tatsache des Fehlens als Deprivation, während Mack und Lansley das Fehlen von Items nur dann als Indikatoren für Deprivation verwenden, wenn dieses Fehlen durch mangelnde Ressourcen erzwungen ist. Damit begegnen sie der u.a. von ihnen und Piachaud (1981) an Townsends Vorgehen kritisierten fehlênden Berlicksichtigung unterschiedlicher individueller Präferenzen. 
Tabelle 3 Korrelation unterschiedlicher Deprivationsindizes.

\begin{tabular}{|c|c|c|c|c|c|c|c|c|c|}
\hline & \multirow{2}{*}{\multicolumn{3}{|c|}{ Ungewichtet }} & \multicolumn{4}{|c|}{$\begin{array}{l}\text { Gewichtet mit der anteiligen } \\
\text { Notwendigkeitsbewertung }\end{array}$} & \multirow{2}{*}{\multicolumn{2}{|c|}{$\begin{array}{l}\text { Gewichtet mit der } \\
\text { Merkmalsverbreitung } \\
\text { Gesamte "Referenz- } \\
\text { Stichprobe gruppen" }\end{array}$}} \\
\hline & & & & \multicolumn{2}{|c|}{$\begin{array}{l}\text { Gesamte } \\
\text { Stichprobe }\end{array}$} & \multicolumn{2}{|c|}{$\begin{array}{l}\text {,Referenz- } \\
\text { gruppen" }\end{array}$} & & \\
\hline & $f_{\text {alle }}$ & $I_{A}$ & $I_{B}$ & $\|_{A}$ & $\|_{B}$ & $\|_{A}$ & \|\|$_{B}$ & IV & V \\
\hline$I_{\text {alle }}$ & 1 & & & & & & & & \\
\hline$I_{A}$ & 0,974 & 1 & & & & & & & \\
\hline$I_{B}$ & 0,775 & 0,801 & 1 & & & & & & \\
\hline$\|_{A}$ & 0,990 & 0,991 & 0,834 & 1 & & & & & \\
\hline $\mathbb{H}_{B}$ & 0,963 & 0,975 & 0,897 & 0,989 & 1 & & & & \\
\hline\|\|$_{A}$ & 0,987 & 0,990 & 0,832 & 0,999 & 0,988 & 1 & & & \\
\hline\|\|$_{B}$ & 0,958 & 0,972 & 0,894 & 0,985 & 0,996 & 0,985 & 1 & & \\
\hline IV & 0,996 & 0,983 & 0,797 & 0,994 & 0,975 & 0,992 & 0,970 & 1 & \\
\hline v & 0,986 & 0,977 & 0,797 & 0,988 & 0,971 & 0,986 & 0,967 & 0,993 & 1 \\
\hline
\end{tabular}

Anmerkung: $n: 3.106$, Erläuterung der Deprivationsindizes im Text

Quelle: Sozialwissenschaftenbus III/96, gewichtete Berechnung

thoden - eher geringen soziodemographischen Unterschiede in der Notwendigkeitsbewertung vermuten, daß es ebenfalls keinen bedeutsamen empirischen Unterschied macht, ob subgruppenspezifisch oder mit dem einfachen Bevölkerungsdurchschnitt gewichtet wird.

Alle hier behandelten Deprivationsindizes lassen sich als (gewichtete) Summe der für jede Person i $(i=1, \ldots, n)$ aus finanziellen Gründen fehlenden Lebensstandardmerkmale ${ }^{9}$ ausdrücken:

$$
\text { INDEX; }=\sum_{j=1}^{K} g_{j i} * X_{j i}
$$

mit: $\mathrm{x}_{\mathrm{ji}}=1$ wenn das Merkmal $\mathrm{j}(\mathrm{j}=1, \ldots, \mathrm{K} ; \mathrm{K}=$ Anzahl der zu berücksichtigenden Items) für Person $i$ aus finanziellen Gründen fehlt und $\mathrm{x}_{\mathrm{ji}}=0$ wenn es verfügbar ist.

$\mathrm{g}_{\mathrm{ji}}=$ das Gewicht, mit dem das Merkmal in den Index eingeht.

Tabelle 3 zeigt die Korrelation mehrerer Deprivationsindizes untereinander. Neben drei Varianten eines einfachen ungewichteten Summenindex $\left(g_{\mathrm{ji}}\right.$ $=1$ für alle $\mathrm{i}$ und $\mathrm{j}$ ) wurden unterschiedliche Versionen von gewichteten Indizes berechnet. Die

${ }^{9}$ Bei den Arbeitsplatzmerkmalen und den Infrastrukturitems wurde aus naheliegenden Gründen nicht nach finanziellen Gründen und anderen Gründen für das Fehlen unterschieden. Bei diesen Merkmalen geht das einfache Fehlen als Indikator für Deprivation in die berechneten Indizes ein. drei ungewichteten Indizes unterscheiden sich lediglich in der Auswahl der berücksichtigten Items: Index $\mathrm{I}_{\text {alle }}$ umfaßt (wie auch die im Anschluß beschriebenen gewichteten Indizes) alle 42 erfragten Items. Demgegenüber berücksichtigt Index $\mathrm{I}_{\mathrm{A}}$ lediglich die Untermenge von Items, die von einer Mehrheit der Befragten zum notwendigen Lebensstandard gerechnet werden (vgl. Spalte A von Tabelle 1) und Index $I_{B}$ lediglich solche, die von einer Mehrheit der Befragten zum minimal notwendigen Lebensstandard gerechnet werden (Tabelle 1, Spalte B). Diese Indizes stellen also die beiden mit den hier analysierten Daten möglichen Implementierungen des Vorgehens von Mack und Lansley dar.

In Anlehnung an den Vorschlag von Halleröd (1995) wurden vier Indizes berechnet, bei denen das Fehlen der Merkmale nicht mit einem Gewicht von 1 , sondern mit einem aus der anteiligen Notwendigkeitsbewertung abgeleiteten Wert berücksichtigt wurde. Bei den Indizes $\mathrm{II}_{\mathrm{A}}$ und $\mathrm{II}_{B}$ entspricht der Gewichtungsfaktor dabei dem Anteil von Befragten in der gesamten Stichprobe, die das jeweilige Merkmal zum notwendigen $\left(\mathrm{II}_{\mathrm{A}}\right)$ bzw. zum minimal notwendigen Lebensstandard $\left(\mathrm{II}_{B}\right)$ rechnen. Die Indizes $\mathrm{III}_{\mathrm{A}}$ und $\mathrm{III}_{\mathrm{B}}$ verwenden bei ansonsten analoger Berechnungsweise die Notwendigkeitsbewertungen in der, Referenzgruppe der Befragten. Hierzu wurden insgesamt 16 Referenzgruppen nach folgenden Merkmalen gebildet: Region (Ost-West), Bildung (Hauptschulabschluß/ 
kein Abschluß und mittlere Reife oder höher) sowie vier Altersklassen in der auch in Tabelle 2 verwendeten Klassierung. Die Gewichte für die verbleibenden beiden Indizes bestimmen sich aus dem Anteil der Befragten insgesamt (IV) bzw. in der Referenzgruppe (V) die über das jeweilig Merkmale verfügen.

Diese extrem hohen Korrelationen bedürfen m.E. kaum eines weiteren Kommentares. Zumindest bei der hier gewählten Einteilung von Referenzgruppen und mit den vorliegenden Daten macht es keinen Unterschied ob mit der anteiligen Notwendigkeitsbewertung oder mit der Verbreitung der erfragten Items gewichtet wird. Ebenso ist es aus der Sicht des Endergebnisses bedeutungslos, ob Referenzgruppen berücksichtigt werden oder nicht. Selbst der völlige Verzicht auf jede Form der Gewichtung führt zu einem Index, der mit den anderen Varianten derart hoch korreliert, daß für die Anwendung in der Armutsforschung mit identischen Ergebnissen zu rechnen ist. Weiterhin macht es bei Indizes die auf allen erfragten Items basieren keinen nennenswerten Unterschied ob die Notwendigkeitsbewertung in der breiteren Operationalisierung als notwendiger Lebensstandard oder enger als minimal notwendiger Lebensstandard zu Bestimmung von Gewichtungsfaktoren herangezogen wird. Einschränkend ist hier allenfalls anzumerken, daß der ungewichtete Index $\left(I_{a l}\right.$ le) in dieser Form auch Items enthält, die nicht von einer Mehrheit der Befragten zum notwendigen Lebensstandard gerechnet werden. Bei einer - im Einklang mit dem Vorschlag von Mack und Lansley (1985) notwendigen - Verkürzung des Index nimmt auch die Korrelation mit den anderen ab. Das gilt insbesondere dann, wenn man nur solche Merkmale berilcksichtigt, die von einer Mehrheit zum unbedingt notwendigen Lebensstandard gerechnet werden $\left(I_{B}\right)$.

Für einen Deprivationsindex nach dem Vorschlag Muffels (1993) konnte Lipsmeier (1995: 97) mit Daten einer anderen deutschen Umfrage ebenfalls eine Korrelation von 0,98 zwischen einem mit und einem ohne Berücksichtigung von Referenzgruppen berechneten Index nachweisen. Auch Halleröd et al. (1997: 222ff) berichten ein sehr hohes $\mathrm{Ma} B$ an Überlappung zwischen einem (ungewichteten, auf einer Teilmenge der Items basierenden) Index nach dem Vorschlag von Mack und Lansley und dem von ihnen vorgeschlagenen, referenzgruppenspezifisch mit der anteiligen Notwendigkeitsbewertung gewichteten Index.

\section{Fazit}

Die wichtigsten Ergebnisse dieses Beitrages lassen sich unter vier Punkten zusammenfassen: 1 . Es läßt sich ein Kern von Lebensstandardmerkmalen ausmachen, für die ein so hohes $\mathrm{Ma} B$ an Übereinstimmung der Notwendigkeitsbewertungen feststellbar ist, $\mathrm{daB}$ von einem faktischen Konsens über deren Zugehörigkeit zu einer für Deutschland als Minimum akzeptablen Lebensweise ausgegangen werden kann. 2. Das Antwortverhalten der Befragten zeigt schlüssige Hinweise darauf, $\mathrm{da} \beta$ mit der gewählten Fragestellung im wesentlichen normative Urteile über den notwendigen Lebensstandard erhoben werden. 3. Unter Verwendung multivariater Verfahren zeigen sich kaum wesentliche Unterschiede in den Bewertungen verschiedener Teilgruppen der Gesellschaft. Schließlich konnte gezeigt werden, daß es (4.) empirisch keinen nennenswerten Unterschied macht, ob bei der Zusammenfassung von Deprivationserscheinungen zu aggregierten Maßen subgruppenspezifische Bewertungen herangezogen werden oder nicht.

Zusammengenommen sind diese Ergebnisse von zentraler Bedeutung für die Messung von Armut mit den hier verwendeten Methoden. Sie zeigen, $\mathrm{da} B$ entsprechende Ansätze auch auf deutsche Verhältnisse übertragbar sind. Bei allen theoretischen Argumenten für die Bedeutung von Referenzgruppen kann nicht übersehen werden, $\mathrm{da} B$ zumindest eine einfache Implementierung über einige soziodemographische Variablen kaum einen Erkenntnisgewinn bietet. Die Verwendung unterschiedlicher Gewichtungsfaktoren führt somit, insbesondere wenn diese subgruppenspezifisch bestimmt werden, - ohne wirklichen Nutzen - zu einer erheblichen Reduzierung der Anschaulichkeit des Deprivationsindex von Mack und Lansley (1985). Dennoch sollten die theoretischen Argumente für eine unterschiedliche Bedeutung einzelner Items für den Lebensstandard von Menschen in unterschiedlichen Lebenssituationen Anlaß für weitere Forschungen sein. Anknüpfungspunkte hierfür wären zum einen die theoriegeleitete Suche nach soziologisch relevanten Referenzgruppen und zum anderen der von Nolan und Whelan (1996: 87ff) vorgeschlagene Verzicht auf einen einheitlichen (auf den gleichen Items basierenden) Deprivationsindex für alle Befragten.

Obwohl nach den hier berichteten Ergebnissen viel dafür spricht, die Bewertungen von Befragten für die empirisch gestützte Bestimmung von Min- 
deststandards heranzuziehen, sollte nicht übersehen werden, daß den Armutsforschern und -forscherinnen damit keineswegs alle (normativen) Entscheidungen abgenommen sind. Dieses wurde z. B. bei der Abgrenzung zwischen dem minimal notwendigen Lebensstandard und dem notwendigen Lebensstandard deutlich. Ein weiteres (hier nicht thematisiertes) Beispiel ist die Bestimmung von Schwellenwerten von Deprivationsindizes, ab denen von genügender empirischer Evidenz für einen Ausschluß von der in einer Gesellschaft als Minimum annehmbaren Lebensweise und damit für das Vorliegen von Armut - auszugehen ist. Demgegenüber scheinen einige der in der Einleitung aufgeworfenen Entscheidungsprobleme, wie z.B. die Entscheidung für eine bestimmte Form der Indexbildung, weniger gravierende Folgen für das empirische Bild von Deprivation zu haben, als man auf Grund der von den jeweiligen Autoren vorgebrachten Argumente vermuten könnte.

\section{Literatur}

Alves, W.M./ Rossi, P.H., 1978: Who Should Get What? Fairness Judgements of the Distribution of Earnings. American Journal of Sociology 84: 541-564.

Andreß, H.-J., 1994: Pretest einer Skala zur Messung subjektiver Deprivation. Bielefeld: Universität Bielefeld, Fakultät für Soziologie, Arbeitspapier Nr. 14 des DFGProjektes „Versorgungsstrategien privater Haushalte im unteren Einkommensbereich (VuE)".

AndreB, H.-J./Lipsmeier, G., 1995: Was gehört zum notwendigen Lebensstandard und wer kann ihn sich leisten? Ein neues Konzept zur Armutsmessung. Aus Politik und Zeitgeschichte B 31-32/95: 35-49.

Andreß, H.-J./Burkatzki, E./Lipsmeier, G./Salentin, K./ Schulte, K./Strengmann-Kuhn, W., 1996: Leben in Armut. Analysen der Verhaltensweisen armer Haushalte mit Umfragedaten. Bielefeld: Universität Bielefeld, Fakultät für Soziologie, Endbericht des DFGProjektes "Versorgungsstrategien privater Haushalte im unteren Einkommensbereich (VuE)“.

Desai, M./Shah, A., 1988: An Econometric Approach to the Measurement of Poverty. Oxford Economic Papers 40: 505-522.

Dickinson, J., 1991: Values and judgements of wage differentials. British Journal of Social Psychology 30: 267270.

Dornstein, M., 1989: The fairness judgements of received pay and their determinants. Journal of Occupational Psychology 62: 287-299.

Europäische Gemeinschaften, 1985: Beschluß des Rates vom 19.Dezember 1984 uber gezielte Maßnahmen zur Bekämpfung der Armut auf Gemeinschaftsebene. Amtsblatt der Europäischen Gemeinschaften, Abl. Nr. L 2: 24-25.
GFM-Getas/WBA (Hrsg.), 1997: Sozialwissenschaftenbus III/96 - Methodendokumentation zur technischen Organisation und Durchführung. Hamburg: GFM-Getas.

Goedhart, T./Halberstadt, V./Kapteyn, A./van Praag, B., 1977: The Poverty Line: Concept and Measurement. Journal of Human Resources XII: 503-520.

Gordon, D./Pantazis, C., 1997a: The Publics Perception of Necessities and Poverty. S. 71-96 in:D. Gordon/C. Pantazis (Hrsg.): Breadline Britain in the 1990s. Aldershot et al.: Ashgate.

Gordon, D./Pantazis, C. (Hrsg.), 1997b: Breadline Britain in the 1990 s. Aldershot et al.: Ashgate.

Hagenaars, A.J.M., 1986: The Perception of Poverty. Amsterdam: North-Holland Publishing Co.

Halleröd, B., 1994: Poverty in Sweden: A New Approach to Direct Measurement of Consensual Poverty, Umea Studies in Sociology No. 106. Umea: University of Umea.

Halleröd, B., 1995: Making ends meet: perceptions of poverty in Sweden. Scandinavian Journal of social welfare 4: 174-189.

Halleröd, B./Bradshaw, J./Holmes, H., 1997: Adapting the consensual definition of poverty. S. 213-234 in: D. Gordon/C. Pantazis (Hrsg.): Breadline Britain in the 1990s. Aldershot et al.: Ashgate.

Hochschild, J.L., 1981: What's Fair? American Beliefs about Distributive Justice. Cambridge: Harvard University Press.

Kalton, G., 1983: Introduction to Survey Sampling. Sage University Paper series on Quantitative Applications in the Social Sciences, No. 07-035. Newbury Park et al.: Sage.

Kangas, O./Ritakallio, V.-M., 1998: Different Methods Different Results. Approaches to multidimensional Poverty. S. 167-203 in: H.-J. Andreß (Hrsg.): Empirical Poverty Research in a Comparative Perspective. Aldershot: Ashgate.

Kanning, P./Mummendey, A., 1993: Soziale Vergleichsprozesse und die Bewältigung 'negativer sozialer Identität' - Eine Feldstudie in Ostdeutschland. Zeitschrift für Sozialpsychologie: 211-217.

Kapteyn, A./Wansbeck, T., 1982: Empirical Evidence on Preference Formation. Journal of Economic Psychology 2: 137-154.

Kapteyn, A./van Praag, B.M.S./van Herwaarden, F.G., 1978: Individual Welfare Functions and Social Reference Spaces. Economics Letters 1: 173-177.

Korn, E.L./Graubard, B.I., 1990: Simultaneous Testing of Regression Coefficients With Complex Survey Data: Use of Bonferoni t Statistics. The American Statistician 44: $270-276$.

Lamm, H./Schwinger, T., 1980: Norms concerning Distributive Justice: Are Needs Taken into Consideration in Allocation Decisions? Social Psychology Quarterly 43: 425-429.

Lipsmeier, G., 1995: Zur Messung von Armut: Das Konzept der subjektiven Deprivation. Eine empirische Betrachtung mit Umfragedaten. Bielefeld: Universität Bielefeld, Fakultät für Soziologie, unveröffentlichte Diplomarbeit. 
Mack, J./Lansley, S., 1985: Poor Britain. London: Allen \& Unwin.

Muffels, R.J.A., 1993: Welfare Economic Effects of Social Security. Essays on Poverty, Social Security and Labour Market: Evidence from Panel Data. Tilburg: KUB.

Muller, H.-P., 1992: Sozialstruktur und Lebensstile. Der neuere theoretische Diskurs uber soziale Ungleichheit. Frankfurt am Main: Suhrkamp.

Nolan, B./Whelan, C.T., 1996: Resources, Deprivation and Poverty. Oxford: Clarendon Press.

Piachaud, D., 1981: Peter Townsend and the Holy Grail. New Society, 10.09.: 419-421.

Rokeach, M., 1973: The Nature of Human Values. New York: The Free Press

Spellerberg, A., 1996: Lebensstile, soziale Lage und Wohlbefinden. S. 205-221 in: W. Zapf/R. Habich (Hrsg.): Wohlfahrtsentwicklung im vereinten Deutschland. Berlin: Sigma.

Stata Corporation, 1997: Stata Statistical Software Release 5.0, Reference P-Z. College Station TX: Stata Corporation
Townsend, P., 1979: Poverty in the United Kingdom. Berkeley and Los Angeles: University of California Press

van den Bosch, K., 1998: Perceptions of the minimal standard of living in Belgium: Is there a consensus? S. 135166 in: H.-J. Andreß (Hrsg.): Empirical Poverty Research in a Comparative Perspective. Aldershot: Ashgate.

Veit-Wilson, J., 1998: Setting Adequacy Standards. How Governments define minimum Incomes. Bristol: The Policy Press.

Walker, R., 1987: Consensual Approaches to the Definition of Poverty: Towards an Alternative Methodology. Journal of Social Policy 16: 213-226.

Wegener, B./Liebig, S., 1993: Eine Grid-Group-Analyse Sozialer Gerechtigkeit. Die neuen und alten Bundesländer im Vergleich. Kölner Zeitschrift für Soziologie und Sozialpsychologie 45: 668-690.

Whelan, B.J., 1993: Non-monetary indicators of poverty. S. 24-42 in: J. Berghman/B. Cantillon (Hrsg.): The european face of social security. Essays in the Honour of Herman Deleeck. Aldershot: Avebury. 